\title{
Outage Probability Analysis for the Multi-Carrier NOMA Downlink Relying on Statistical CSI
}

\author{
Shenhong Li, Student Member, IEEE, Mahsa Derakhshani Member, IEEE, Sangarapillai Lambotharan, Senior \\ Member, IEEE, Lajos Hanzo, Fellow, IEEE
}

\begin{abstract}
In this treatise, we derive tractable closed-form expressions for the outage probability of the single cell multicarrier non-orthogonal multiple access (MC-NOMA) downlink, where the transmitter side only has statistical CSI knowledge. In particular, we analyze the outage probability with respect to the total data rates (summed over all subcarriers), given a minimum target rate for the individual users. The calculation of outage probability for the distant user is challenging, since the total rate expression is given by the sum of logarithmic functions of the ratio between two shifted exponential random variables, which are dependent. In order to derive the closed-form outage probability expressions both for two subcarriers and for a general case of multiple subcarriers, efficient approximations are proposed. The probability density function (PDF) of the product of shifted exponential distributions can be determined for the near user by the Mellin transform and the generalized upper incomplete Fox's H function. Based on this PDF, the corresponding outage probability is presented. Finally, the accuracy of our outage analysis is verified by simulation results.
\end{abstract}

Index Terms-Outage probability, Non-orthogonal multiple access, MC-NOMA, Channel state information (CSI), Shifted exponential distribution, Mellin transform, Generalized upper incomplete Fox's $\mathrm{H}$ function

\section{INTRODUCTION}

Non-orthogonal multiple access (NOMA) has been recently introduced as a potential technique of improving the spectral efficiency for next generation systems beyond that of orthogonal multiple access (OMA) [1], [2]. As opposed to OMA schemes, NOMA is capable of supporting a large number of users via non-orthogonal resource allocation by the simultaneous exploitation of the time, frequency and code domains as well as multiplexing them at different power levels [3], [4], [5]. The superposition of signals is performed by ensuring that the inter-user interference can be successfully removed by applying successive interference cancellation (SIC) at the receiver. The authors of [4], [6], [7] introduced the concepts, challenges, applications and research trends of NOMA systems. The authors of [4] introduced the basics of NOMA,

S. Li, M. Derakhshani, and S. Lambotharan are with the Signal Processing and Networks Research Group, Wolfson School, Loughborough University, Leicestershire, LE11 3TU, United Kingdom (email: s.li3@lboro.ac.uk; m.derakhshani@lboro.ac.uk; s.lambotharan@lboro.ac.uk).

L. Hanzo is with the School of Electronics and Computer Science, University of Southampton, Southampton, SO17 1BJ, United Kingdom (email: hanzo@soton.ac.uk).

This work was supported in part by the Engineering and Physical Sciences Research Council under Grant EP/R006385/1, EP/Noo4558/1, EP/PO34284/1, COALESCE, of the Royal Society's Global Challenges Research Fund Grant as well as of the European Research Council's Advanced Fellow Grant QuantCom. including their power allocation policies, the application of SIC, and compared NOMA and conventional OMA. This study also discussed the recent developments in NOMA, such as the combination of NOMA and MIMO schemes, cooperative NOMA, and NOMA in cognitive radio networks. Finally, research challenges and promising future directions were provided. The authors of [6] provided a comprehensive review of power-domain NOMA and discussed its performance with the aid of numerical results. In [7], the authors highlighted the basic principles, key features, receiver complexity and engineering feasibility of NOMA systems.

The achievable performance of NOMA systems significantly relies on the accuracy of the channel state information (CSI). Therefore, the outage probability of failing to support a minimum target rate is a critical performance metric. The outage analysis of NOMA has received considerable research attention both under the assumptions of only having statistical CSI $^{1}$ (e.g., [8]-[12]), imperfect CSI (e.g., [13], [14]), and also imperfect SIC (e.g., [15], [16]). These studies have been focused mainly on single-carrier NOMA systems. Hence, to solve this open problem, we focus on the outage analysis of the multi-carrier NOMA downlink in the face of only having statistical knowledge of the CSI available at the transmitter. Furthermore, having the exact knowledge of the instantaneous CSI at the transmitter constitutes a strong idealized simplifying assumption. Hence, the main motivation of this treatise is to analyze the outage probability w.r.t the data rates, (summed over all subcarriers) in the multi-carrier NOMA downlink under a more realistic model. The outage probability of multicarrier NOMA systems has been studied by ensuring that the outage probability associated with a certain minimum data rate of each user on each subcarrier does not exceed a certain tolerable maximum value. By contrast, in this work, we define and analyze the outage probability in terms of the total data rate of each user under the assumption that channel coding is employed and thus the decoding is performed after combining the signals gleaned from all subcarriers. Assuming that the forward error correction (FEC) codewords extend over a single block, the outage probability for a given rate is the probability that the mutual information of these parallel channels falls (sum rate here) below that rate [17]. In such a case, outage (or failure in decoding) would occur when the detection errors over different subcarriers cannot be identified and corrected, which is the case if the sum rate is lower than the target.

\footnotetext{
${ }^{1}$ Statistical CSI represents the long-term channel state information, which means that only the distribution of the channel gain (including its type and its statistical properties) is known, but not the actual realization.
} 
Moreover, we focus our attention on the case when only the CSI statistics are known at the transmitter and thus an outage event may happen when user $k$ is unable to detect either its own message or the messages of the weaker users for SIC purposes.

\section{A. Literature review}

Previously, to explore the potential gains of applying NOMA, many contributions on NOMA systems assumed that perfect CSI knowledge is available at both the receiver and transmitter sides [3], [18]-[21]. The authors of [18] conceived resource allocation algorithm for full-duplex multicarrier NOMA (MC-NOMA) systems. As a further development, the authors of [19]-[21] investigated dynamic power allocation, optimal resource allocation (power allocation and user pairing) as well as distributed power control in a multicell NOMA system, respectively. However, having the exact knowledge of instantaneous CSI at the transmitter is an idealized simplifying assumption, since finite-delay, finiteaccuracy quantized feedback links must be used. Hence, a more realistic research trend has emerged, focusing on NOMA when only imperfect knowledge of the CSI is available. For example, the authors of [22] studied the power allocation problem of the NOMA downlink in the face of imperfect CSI, while the authors of [13] improved the energy efficiency of a downlink NOMA single-cell network by considering imperfect CSI. They assumed a channel estimation error model where the BS only knows the estimated channel gain and has $a$ priori knowledge of the variance of the estimation error. In these scenarios, the outage probability is an important metric, which is widely used for performance analyses, especially when the communication channel is characterized by random parameters. The existing contributions exploring the outage analysis of NOMA mainly focus on single-carrier scenarios.

As for the single-carrier NOMA downlink, Ding et al. [23] first derived the outage probability for the downlink of a NOMA system in conjunction with the idealized simplifying assumption of perfect channel state information (CSI), assuming that all mobile users were uniformly distributed within a cell. This work provided a closed-form expression of the outage probability, under the limitation of a single-carrier case. Later Hou et al. [9] characterized the outage performance of the downlink in a NOMA system with perfect CSI and fixed power allocation for transmission over Nakagami-m fading channels. Again, this work is also limited to the perfect CSI scenario and single-carrier systems. Timotheous and Krikidis [24] studied the power allocation problem from the viewpoint of fairness under full CSI and average CSI knowledge by applying outage probability (OP) constraints. The effect of imperfect CSI and of second order statistics (SOS)-based CSI on the performance of the NOMA downlink was studied by Yang et al. [25]. By contrast, Shi et al. [26] investigated the outage performance of the downlink in a NOMA system with only statistical CSI knowledge at the transmitter. They derived the optimal power allocation factor in closed form and determined the optimal detection order to avoid excessive outage probabilities. Then Wang et al. [27] also analyzed the outage performance of the downlink in a NOMA system with only statistical CSI knowledge at the transmitter. Yang et al. [28] derived the outage probability for the paired users of a dynamic power allocation based hybrid NOMA systems. Yue et al. [29] also investigated the outage performance of a unified NOMA framework by invoking stochastic geometry. Finally, Cui et al. [30] applied multiple input and multiple output (MIMO) techniques to a NOMA system and analysed the outage probability by considering both the channel's distribution information (CDI) and the channel estimation uncertainty.

As for the single-carrier NOMA uplink, its outage analysis was disseminated in [31]-[33] under statistical CSI, but the analysis was limited to at most three users. In [10], Liu et al. extended the outage analysis of the single-carrier NOMA uplink to an arbitrary number of users. Furthermore, Wang et al. [11] analyzed the outage probability of NOMA for independent but not identically distributed (i.ni.d) Rayleigh fading environments. A closed-form outage expression was derived for both the dynamic and the fixed detection order. Xia et al. [8] proposed an advanced multiuser SIC detector for the NOMA uplink and analyzed the outage performance of this scenario.

The NOMA techniques have evolved from single-carrier NOMA (SC-NOMA) to multi-carrier NOMA (MC-NOMA) [34]. In order to fully realize the potential of NOMA, efficient scheduling techniques are also needed. In conventional multicarrier systems, the total bandwidth is partitioned into multiple subcarriers and each subcarrier is allocated to a single user in order to avoid multiuser interference. Sun et al. [35] have demonstrated that the MC-NOMA provides an excellent system performance compared to the MC-OMA.

However, as for the multi-carrier NOMA downlink, there is paucity of research contributions focusing on its outage analysis. The current approach is to ensure that the outage probability associated with the given data rate of each user on each subcarrier does not exceed a certain value. For instance, Wei et al. [36] proposed a power-efficient resource allocation scheme for MC-NOMA relying on imperfect CSI, while Tweed et al. [16] studied outage constrained resource allocation in conjunction with imperfect SIC, but both used the outage per subcarrier. However, it is not practically attractive to have a specific outage probability for the data rate on each subcarrier, since the ultimate aim of MC-NOMA is to ensure achieving a specific total rate for each user. Hence, in this paper, we consider a more realistic MC-NOMA downlink system where the outage probability is defined in terms of the total data rate of each user averaged over all subcarriers being below a certain threshold. We also examine the outage performance under statistical CSI provision.

\section{B. Contributions}

The key novelty in our work is that of deriving closed-form expressions for the outage probability of multi-carrier NOMA downlink for an arbitrary number of users and subcarriers. Indeed, the outage probability relying on statistical properties has been studied before, but not by unveiling the intricacies of multi-carrier NOMA. To the best of our knowledge, this work 
is the first one to analyze outage in the MC-NOMA downlink, where the outage probability of decoding the transmission of user $k$ at any receiver (at user $k$ or other users closer to the BS) is defined based on the sum rate over all subcarriers as the probability that the transmission of user $k$ cannot be decoded with negligible error probability. There are only a few studies on the OP analysis of MC-NOMA [16], [34], where the OP was defined as the probability of failing to satisfy the minimum required data rate of each user on each subcarrier. By contrast, the OP of each user is defined here as the probability of failing to detect its own message or the messages of the weaker users based on the total rate of an individual user over all subcarriers. As NOMA is more attractive for the case of two users per subcarrier [34], we first consider the case for two users at each subcarrier and analyse the outage probability of both the users who share multiple subcarriers using NOMA. Then, we extend the results to a more general case of an arbitrary number of users. Our main contributions are summarized as follows:

1) We analyze the OP of the far user of the multi-carrier NOMA downlink. The challenge is then to obtain the statistics required for the OP analysis, where the total rate expression is given by the sum of logarithmic functions of the ratio between two shifted exponential random variables, which are dependent. In order to derive a closedform solution, first we transform the sum of logarithmic functions into the product of the ratio between two shifted exponential random variables and then approximate the product by ignoring the high-order terms. The simulation results show that the approximation is quite tight, hence it was used for our subsequent derivations. Then, in the case of two subcarriers, we analytically calculate the PDF and CDF of the inverse shifted exponential random variables, and derive the corresponding OP expression. For the general case of an arbitrary number of subcarriers, the sum of inverse shifted exponential random variables may be accurately modelled by a Gamma distribution. Hence the corresponding closed-form expression is provided for the OP.

2) We also investigate the OP of the near user. We consider that an outage event may happen if the near user cannot detect either its own message or the message of the far user. Thus, first the probability of successfully detecting this user and the probability of successfully detecting the far user are studied. The former is characterized by the complementary CDF (CCDF) of the product of shifted exponential random variables in the case of successful SIC detection. To obtain this CCDF, by applying the Mellin transform [35], we determine the PDF of the product of shifted exponential random variables, which is represented in the form of the generalized upper incomplete Fox's H function. Subsequently, to derive the closed-form OP expression of the near user, we analyze the integral of the generalized upper incomplete Fox's $\mathrm{H}$ function. The Mathematica programming code is also provided for implementing the generalized upper incomplete Fox's $\mathrm{H}$ function. Our simulation results confirm the accuracy of the OP expression derived.

3) We extend the OP analysis to the case of an arbitrary number of users in each subcarrier and derive the OP expression of each user.

4) We compare the OP of each user in our MC-NOMA system to that of an OMA system, which confirmed that our MC-NOMA indeed outperforms the corresponding OMA system.

\section{Organisation}

The remainder of this paper is organized as follows. Section III presents the system model. In Section IV, we study the OP for the minimum rate of two individual users. We extend the OP for the general case of multiplexing an arbitrary number of users per subcarrier in Section V. Simulation results are presented in Section VI, followed by our conclusions in Section VII.

\section{SySTEM MODEL}

Consider a downlink NOMA system supporting $K$ singleantenna users by a single-antenna base station (BS). The channel's power gain between the BS and user $k$ at subcarrier $n$ is formulated by $h_{k, n}=\chi_{k, n} d_{k}^{-\lambda}$, where $\chi_{k, n}$ denotes the squared magnitude of channel coefficient that is assumed to undergo small scale fading, $d_{k}$ is the distance between the $k$ th user and the BS, $k=1,2, \ldots, K$, and $\lambda$ is the path loss exponent. We assume Rayleigh fading channels and $\chi_{k, n}$ are i.i.d. exponential random variables with a mean of one, for every user and subcarrier. Without loss of generality, the distances are sorted as $d_{1}>d_{2}>\cdots>d_{k}>\cdots>d_{K}$, so that user 1 is the farthest user from the BS.

Successive interference cancellation (SIC) is utilized at the receiver side (users) to separate the signals. Explicitly, the SIC receiver first decodes the strongest signal by treating all other signals as interference. After this stage, the strongest signal is removed from the composite signal, and the second strongest signal is decoded and canceled and so on, until the weakest signal is decoded. Generally, the SIC detection order is based on the ordered channel gain information. Relying on statistical CSI knowledge, according to [37] and [38], the optimal detection order is determined based on the average channel gains of the users. If various users have different outage probability requirements, detection order should be based on the weighted channel gains as described in [37]. In our work, we consider identical requirement for outage probability, hence SIC detection order can be based on statistical knowledge of the channels at the transmitter, which in our case is based on the distances of users.

In this system, user 1 is the farther user from the BS, hence user 1 attempts coherent detection of its own signal without performing SIC, because the strongest signal has the lowest interference. For user $k$, where $k>1$, at the first step of the SIC process, the signal of user 1 which is the strongest signal is detected and removed. After this, the signal of user 2 is detected and canceled. This iterative process is repeated until the signal of user $k-1$ is detected and canceled. At this point 
user $k$ detects its own signal without the interference of users $1,2, \ldots, k-1$.

Therefore, we can directly formulate the signal-tointerference-plus-noise ratio (SINR) for user $k$ at subcarrier $n$ as

$$
\mathrm{SINR}_{k, n}= \begin{cases}\frac{\beta_{k, n} h_{k, n}}{h_{k, n} \sum_{j=k+1}^{K} \beta_{j, n}+\sigma^{2}} & k \in 1,2, \ldots K \\ \frac{\beta_{K, n} h_{K, n}}{\sigma^{2}} & k=K .\end{cases}
$$

where $\beta_{k, n}$ is the transmit power allocation of user $k$ at subcarrier $n$ and $\sigma^{2}$ is the additive white Gaussian noise (AWGN) power. In addition, we assume that the AWGN noise power is the same for all users assigned to each subcarrier.

\section{Outage Probability Analysis for Two USERS}

We derive tractable OP expressions of the multi-carrier NOMA downlink when only the statistical knowledge of the CSI is available at the transmitter side. We assume user 1 is the far user while user 2 is closer to the BS.

Assuming perfect interference cancellation at the receiver side, based on (1), the data rate of each user in each subcarrier is expressed as

$R_{1, n}=\log \left(1+\frac{\beta_{1, n} h_{1, n}}{\sigma^{2}+\beta_{2, n} h_{1, n}}\right), \quad R_{2, n}=\log \left(1+\frac{\beta_{2, n} h_{2, n}}{\sigma^{2}}\right)$.

Therefore, the total data rate of each user over all the shared subcarriers is expressed as

$$
\begin{aligned}
& R_{1}=\sum_{n=1}^{N} \log \left(1+\frac{\beta_{1, n} h_{1, n}}{\sigma^{2}+\beta_{2, n} h_{1, n}}\right), \\
& R_{2}=\sum_{n=1}^{N} \log \left(1+\frac{\beta_{2, n} h_{2, n}}{\sigma^{2}}\right) .
\end{aligned}
$$

\section{A. Outage Probability of User 1}

Again we define the OP of user 1 as the probability that the total rate of this user is less than a threshold. Consequently, the OP of user 1 over $N$ subcarriers is

$$
\operatorname{Pr}_{1, N}^{\text {out }}=\operatorname{Pr}\left(R_{1} \leq r_{1}\right),
$$

where $r_{1}$ denotes the target data rate of user 1 .

Let us now reformulate the data rate of user 1 by introducing signal power, Rayleigh fading, and path loss coefficients, yielding:

$$
\begin{aligned}
R_{1} & =\sum_{n=1}^{N} \log \left(1+\frac{\beta_{1, n} d_{1}^{-\lambda} \chi_{1, n}}{\sigma^{2}+\beta_{2, n} d_{1}^{-\lambda} \chi_{1, n}}\right) \\
& =\sum_{n=1}^{N} \log \left(\frac{\sigma^{2}+\left(\beta_{1, n}+\beta_{2, n}\right) d_{1}^{-\lambda} \chi_{1, n}}{\sigma^{2}+\beta_{2, n} d_{1}^{-\lambda} \chi_{1, n}}\right) .
\end{aligned}
$$

Upon defining $A_{n}=\left(\beta_{1, n}+\beta_{2, n}\right) d_{1}^{-\lambda}$ and $B_{n}=\beta_{2, n} d_{1}^{-\lambda}$, the data rate of user 1 is expressed as

$$
R_{1}=\sum_{n=1}^{N} \log \left(\frac{\sigma^{2}+A_{n} \chi_{1, n}}{\sigma^{2}+B_{n} \chi_{1, n}}\right)
$$

where $\chi_{1, n}$ follows an exponential distribution with a mean of one. Again, we aim for calculating the OP of user 1 .

Based on (4) and (6), we rewrite the OP of user 1 as

$$
\operatorname{Pr}_{1, N}^{\text {out }}=\operatorname{Pr}\left(\sum_{n=1}^{N} \log \left(\frac{\sigma^{2}+A_{n} \chi_{1, n}}{\sigma^{2}+B_{n} \chi_{1, n}}\right) \leq r_{1}\right) \text {. }
$$

Upon defining $Z_{n}=\frac{A_{n} \chi_{1, n}+\sigma^{2}}{B_{n} \chi_{1, n}+\sigma^{2}}$, the OP can be expressed as

$$
\operatorname{Pr}_{1, N}^{\text {out }}=\operatorname{Pr}\left(\prod_{n=1}^{N} Z_{n} \leq e^{r_{1}}\right)
$$

To calculate (8), we have to find the distribution of $\prod_{n=1}^{N} Z_{n}$, i.e. the product of multiple ratios between two shifted exponential random variables, which are dependent. To derive a tractable expression for the PDF of this product, we propose a novel approximation method for accurately representing $\prod_{n=1}^{N} Z_{n}$. First, let us transform $Z_{n}$ as follows:

$$
Z_{n}=u_{n}-\frac{\sigma^{2} m_{n}}{B_{n} \chi_{1, n}+\sigma^{2}}=m_{n}\left[\frac{u_{n}}{m_{n}}-\frac{\sigma^{2}}{B_{n} \chi_{1, n}+\sigma^{2}}\right],
$$

where $u_{n}=\frac{A_{n}}{B_{n}}$ and $m_{n}=\frac{A_{n}}{B_{n}}-1$. Hence, $\prod_{n=1}^{N} Z_{n}$ can be written as

$$
\prod_{n=1}^{N} Z_{n}=\prod_{n=1}^{N} m_{n} \times \prod_{n=1}^{N}\left[\frac{u_{n}}{m_{n}}-\frac{\sigma^{2}}{B_{n} \chi_{1, n}+\sigma^{2}}\right] .
$$

In (10), $\prod_{n=1}^{N} Z_{n}$ is represented as a product of binomials. As $N$ increases, the product will result in higher-order terms related to the product of the $\frac{\sigma^{2}}{B_{n} \chi_{1, n}+\sigma^{2}}$ terms. This will increase the complexity of computations. Therefore, since $\frac{\sigma^{2}}{B_{n} \chi_{1, n}+\sigma^{2}}$ is smaller than one, we approximate $\prod_{n=1}^{N} Z_{n}$ by removing the higher-order terms, yielding

$$
\prod_{n=1}^{N} Z_{n} \approx \prod_{n=1}^{N} u_{n}-\sum_{n=1}^{N}\left(\prod_{n^{\prime} \neq n} u_{n^{\prime}}\right) \frac{m_{n} \sigma^{2}}{B_{n} \chi_{1, n}+\sigma^{2}} .
$$

To confirm the accuracy of this approximation, we have compared the PDF and CDF of the approximated representation of $\prod_{n=1}^{N} Z_{n}$ in (11) to the original one in (10) under different settings ${ }^{2}$. Figure 1 represents the PDF and CDF results for the cases of both two subcarriers and four subcarriers. It is apparent that the shape of histogram PDF and empirical CDF for the approximation coincides with that of the original ones. Hence, the new approximation method of the product of the ratios between two shifted exponential random variables is considered to be satisfactory for further analyses. Furthermore, we have investigated the sensitivity of the approximation to different parameters, such as $t=\frac{\beta_{1, n}}{\beta_{2, n}}$, $\rho=\frac{d_{1}}{d_{2}}, \mathrm{SNR}_{2, n}=\frac{\beta_{2, n} d_{2}^{-\lambda}}{\sigma^{2}}$ for the case of four subcarriers. Figure 2 demonstrates the CDF results considering different values of $t, \rho, \mathrm{SNR}_{2, n}$ aiming to evaluate the accuracy of $\mathrm{OP}$ approximation under different cases. It is apparent that the approximation is accurate enough in all cases.

${ }^{2}$ Note by means of the properties of downlink NOMA system, the value of $B_{n} \leq A_{n}-B_{n}$, thus, $2 B_{n} \leq A_{n}$ and $B_{n} \leq A_{n}$ 

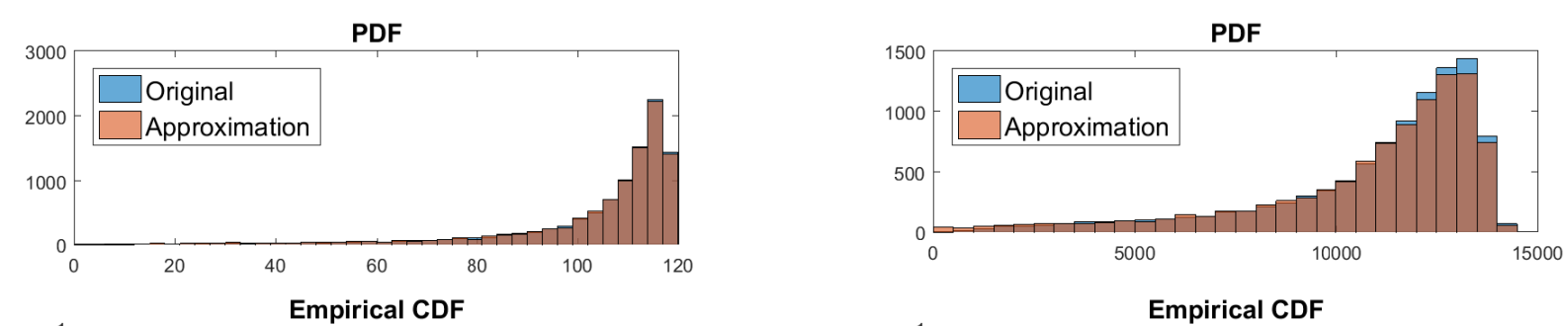

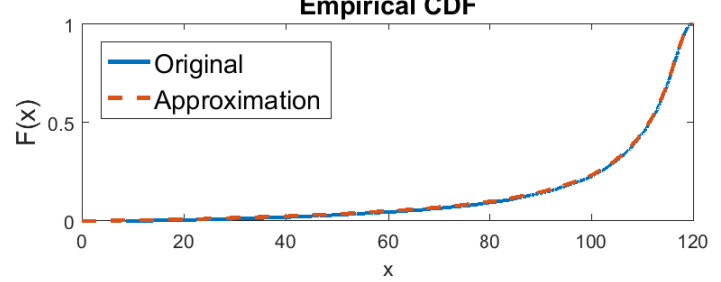

a. Two subcarriers

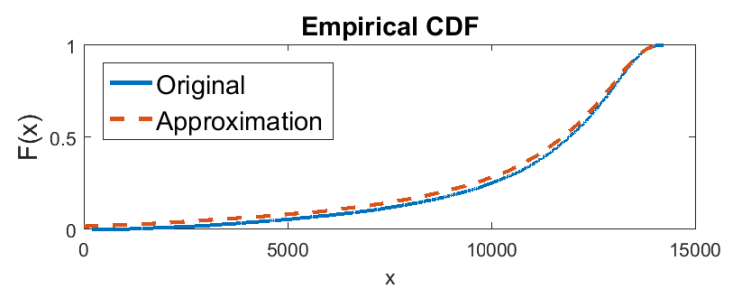

b. Four subcarriers

Fig. 1. The PDF and CDF $\left(\rho=1.5, t=2, \mathrm{SNR}_{2, n}=18 d B\right)$

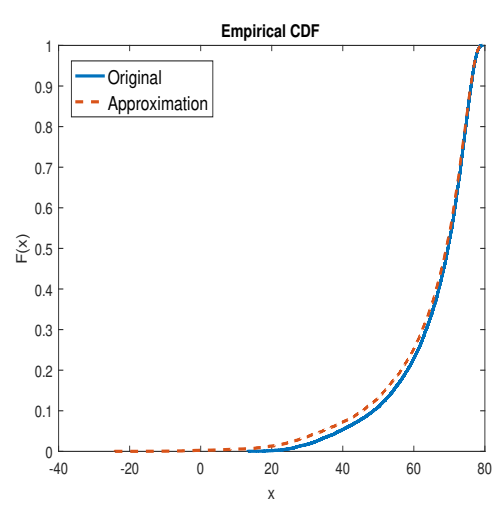

(a) $\rho=1.5, t=2$

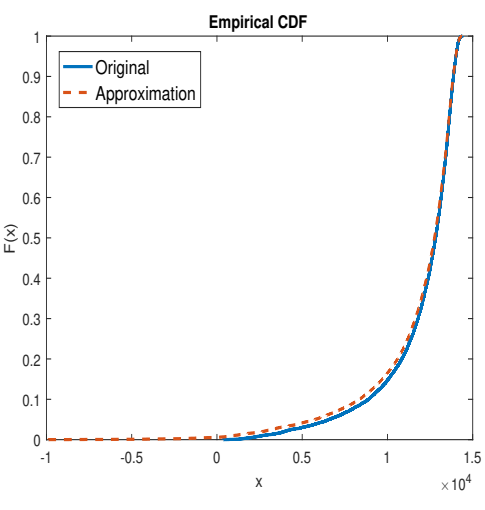

(b) $\rho=1.5, t=10$

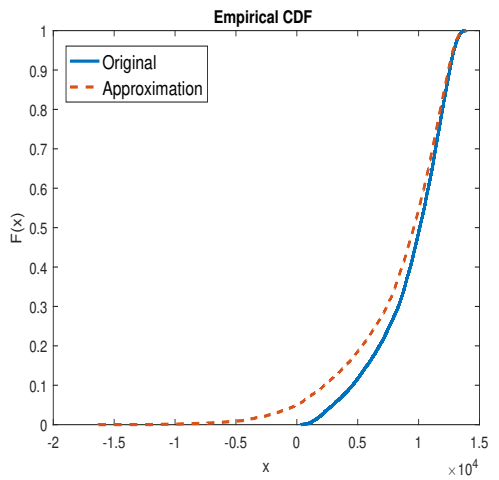

(c) $\rho=2, t=10$

Fig. 2. The Empirical $\mathrm{CDF}\left(\mathrm{SNR}_{2, n}=18 d B\right)$

Having established accuracy of this approximation, we analyze the CDF and PDF of the approximated parameters. Let us define $Y_{n}=\left(\prod_{n^{\prime} \neq n} u_{n^{\prime}}\right) \frac{m_{n} \sigma^{2}}{B_{n} \chi_{1, n}+\sigma^{2}}$. Based on (8) and (11), the OP of user 1 becomes

$$
\begin{aligned}
\operatorname{Pr}_{1, N}^{\text {out }} & =\operatorname{Pr}\left(\prod_{n=1}^{N} u_{n}-\sum_{n=1}^{N} Y_{n} \leq e^{r_{1}}\right) \\
& =\operatorname{Pr}\left(\prod_{n=1}^{N} u_{n}-e^{r_{1}} \leq \sum_{n=1}^{N} Y_{n}\right) .
\end{aligned}
$$

To calculate the OP based on (12), first we derive the probability distribution of $Y_{n}$. Considering that $\prod_{n=1}^{N} u_{n}$ has a fixed value and $\chi_{1, n}$ obeys the exponential distribution, the $\mathrm{CDF}$ and PDF of $Y_{n}$ can be easily calculated. Introducing a new variable $s_{n}=\left(\prod_{n^{\prime} \neq n} u_{n^{\prime}}\right) m_{n}$, we can write $Y_{n}=\frac{\sigma^{2} s_{n}}{B_{n} \chi_{1, n}+\sigma^{2}}$. Therefore, the $\mathrm{CDF}$ of $Y_{n}$ may be expressed as

$$
\begin{gathered}
F_{Y_{n}}\left(y_{n}\right)=\operatorname{Pr}\left(Y_{n} \leq y_{n}\right)=\operatorname{Pr}\left(\frac{\sigma^{2} s_{n}}{B_{n} \chi_{1, n}+\sigma^{2}} \leq y_{n}\right) \\
=1-\operatorname{Pr}\left(\chi_{1, n} \leq \frac{\sigma^{2}\left(s_{n}-y_{n}\right)}{B_{n} y_{n}}\right)
\end{gathered}
$$

$$
= \begin{cases}e^{\frac{\sigma^{2}\left(y_{n}-s_{n}\right)}{B_{n} y_{n}}}, & 0 \leq y_{n} \leq s_{n} \\ 0, & y_{n}<0 \\ 1, & y_{n}>s_{n} .\end{cases}
$$

Consequently, we can calculate the PDF of $Y_{n}$ as

$$
f_{Y_{n}}\left(y_{n}\right)= \begin{cases}e^{\frac{\sigma^{2}\left(y_{n}-s_{n}\right)}{B_{n} y_{n}}} \times \frac{\sigma^{2} s_{n}}{B_{n} y_{n}^{2}}, & 0 \leq y_{n} \leq s_{n}, \\ 0, & \text { otherwise }\end{cases}
$$

Next, based on (13) and (14), we will discuss the OP of different number of subcarriers.

1) The Case of Two Subcarriers: According to (12), for $N=2$, we have

$$
\operatorname{Pr}_{1,2}^{\text {out }}=1-\operatorname{Pr}\left(Y_{1}+Y_{2} \leq C\right),
$$

where $C=u_{1} u_{2}-e^{r_{1}}$. To calculate the OP of user 1 for the case of $N=2$ according to (15), we proceed by deriving the probability of $\operatorname{Pr}\left(Y_{1}+Y_{2} \leq C\right)$ (based on (13) and (14)) and proceed by defining:

$$
\operatorname{Pr}\left(Y_{2} \leq C-y_{1}\right)= \begin{cases}e^{\frac{\sigma^{2}\left(C-y_{1}-s_{2}\right)}{\left(C-y_{1}\right) B_{2}}}, & C-s_{2} \leq y_{1} \leq C, \\ 0, & y_{1}>C, \\ 1, & y_{1}<C-s_{2} .\end{cases}
$$


Subsequently, we can represent $\operatorname{Pr}\left(Y_{1}+Y_{2} \leq C\right)$ based on (16) as

$$
\begin{aligned}
\operatorname{Pr}\left(Y_{1}+Y_{2} \leq C\right)= & \int_{-\infty}^{C-s_{2}} f_{Y_{1}}\left(y_{1}\right) d y_{1} \\
& +\int_{C-s_{2}}^{C} e^{\frac{\sigma^{2}\left(C-y_{1}-s_{2}\right)}{\left(C-y_{1}\right) B_{2}}} f_{Y_{1}}\left(y_{1}\right) d y_{1} .
\end{aligned}
$$

Thus, the probability of $\operatorname{Pr}\left(Y_{1}+Y_{2} \leq C\right)$ consists of two integrations. The upper limit of $\int_{-\infty}^{C-s_{2}} f_{Y_{1}}\left(y_{1}\right) d y_{1}$ is $C-s_{2}$, and $\int_{C-s_{2}}^{C} e^{\frac{\sigma^{2}\left(C-y_{1}-s_{2}\right)}{\left(C-y_{1}\right) B_{2}}} f_{Y_{1}}\left(y_{1}\right) d y_{1}$ is defined over a closed interval $\left[C-s_{2}, C\right]$. In the next step, we determine the probability of $\operatorname{Pr}\left(Y_{1}+Y_{2} \leq C\right)$ by discussing different ranges of $C$ compared to $s_{1}$ and $s_{2}$.

(a) For $C<0$, we have $\operatorname{Pr}\left(Y_{1}+Y_{2} \leq C\right)=0$, because the probability of a positive value being smaller than a negative value is equal to zero.

(b) For $0 \leq C \leq s_{2}$, we have $\operatorname{Pr}\left(Y_{1}+Y_{2} \leq C\right)=$ $\int_{0}^{\min \left(C, s_{1}\right)} e^{\frac{\sigma^{2}\left(C-y_{1}-s_{2}\right)}{\left(C-y_{1}\right) B_{2}}} f_{Y_{1}}\left(y_{1}\right) d y_{1}$

(c) For $s_{2}<C \leq s_{1}+s_{2}$, we have $\operatorname{Pr}\left(Y_{1}+Y_{2} \leq C\right)=$ $\int_{0}^{C-s_{2}} f_{Y_{1}}\left(y_{1}\right) d y_{1}+\int_{C-s_{2}}^{\min \left(C, s_{1}\right)} e^{\frac{\sigma^{2}\left(C-y_{1}-s_{2}\right)}{\left(C-y_{1}\right) B_{2}}} f_{Y_{1}}\left(y_{1}\right) d y_{1}$

(d) For $C>s_{1}+s_{2}$, we have $\operatorname{Pr}\left(Y_{1}+Y_{2} \leq C\right)=1$.

Let us now summarize all the conditions as

$$
\operatorname{Pr}\left(Y_{1}+Y_{2} \leq C\right)=
$$

$$
\begin{cases}0, & C<0, \\ \int_{0}^{\min \left(C, s_{1}\right)} e^{\frac{\sigma^{2}\left(C-y_{1}-s_{2}\right)}{\left(C-y_{1}\right) B_{2}}} f_{Y_{1}}\left(y_{1}\right) d y_{1}, & 0 \leq C \leq s_{2}, \\ \int_{0}^{C-s_{2}} f_{Y_{1}}\left(y_{1}\right) d y_{1}+ & \\ \int_{C-s_{2}}^{\min \left(C, s_{1}\right)} e^{\frac{\sigma^{2}\left(C-y_{1}-s_{2}\right)}{\left(C-y_{1}\right) B_{2}}} f_{Y_{1}}\left(y_{1}\right) d y_{1}, & s_{2}<C \leq s_{1}+s_{2}, \\ 1, & C>s_{1}+s_{2} .\end{cases}
$$

According to (18), to derive an expression for $\operatorname{Pr}\left(Y_{1}+\right.$ $\left.Y_{2} \leq C\right)$ and hence for the OP, we have to calculate $\int e^{\frac{\sigma^{2}\left(C-y_{1}-s_{2}\right)}{\left(C-y_{1}\right) B_{2}}} f_{Y_{1}}\left(y_{1}\right) d y_{1}$. Hence, we discuss the derivation of this integral. First, substituting $f_{Y_{1}}\left(y_{1}\right)$ based on (14) into $\int e^{\frac{\sigma^{2}\left(C-y_{1}-s_{2}\right)}{\left(C-y_{1}\right) B_{2}}} f_{Y_{1}}\left(y_{1}\right) d y_{1}$, we have

$$
\begin{aligned}
& \int e^{\frac{\sigma^{2}\left(C-y_{1}-s_{2}\right)}{\left(C-y_{1}\right) B_{2}}} f_{Y_{1}}\left(y_{1}\right) d y_{1}= \\
& e^{\frac{\sigma^{2}}{B_{1}}} e^{\frac{\sigma^{2}}{B_{2}}} \frac{\sigma^{2} s_{1}}{B_{1}} \int \frac{1}{y_{1}^{2}} e^{\frac{-\sigma^{2} s_{2}}{B_{2}} \frac{1}{C-y_{1}}} e^{\frac{-\sigma^{2} s_{1}}{B_{1}} \frac{1}{y_{1}}} d y_{1} .
\end{aligned}
$$

According to (20), we only have to calculate the integral of $\int y_{1}^{-2} e^{\frac{-a_{2}}{C-y_{1}}} e^{\frac{-a_{1}}{y_{1}}} d y_{1}$ where $a_{1}=\frac{\sigma^{2} s_{1}}{B_{1}}$ and $a_{2}=\frac{\sigma^{2} s_{2}}{B_{2}}$. Since the integral cannot be solved analytically, we evaluate it by numerical integration in Matlab. Therefore, we can express the $\mathrm{OP}$ as

$$
\mathrm{Pr}_{1,2}^{\text {out }}=
$$

$$
\left\{\begin{array}{lc}
1, & C<0 \\
1-e^{\frac{\sigma^{2}}{B_{1}}} e^{\frac{\sigma^{2}}{B_{2}}} \frac{\sigma^{2} s_{1}}{B_{1}} I\left(\min \left(C, s_{1}\right)\right), & 0 \leq C \leq s_{2} \\
1-e^{\frac{\sigma^{2}\left(C-s_{2}-s_{1}\right)}{B_{1}\left(C-s_{2}\right)}}-e^{\frac{\sigma^{2}}{B_{1}}} e^{\frac{\sigma^{2}}{B_{2}}} \frac{\sigma^{2} s_{1}}{B_{1}} I\left(\min \left(C, s_{1}\right)\right) \\
-e^{\frac{\sigma^{2}}{B_{1}}} e^{\frac{\sigma^{2}}{B_{2}}} \frac{\sigma^{2} s_{1}}{B_{1}} I\left(C-s_{2}\right), & s_{2} \leq C \leq s_{1}+s_{2} \\
0, & C>s_{1}+s_{2},
\end{array}\right.
$$

where we have $I(t)=\int_{0}^{t} y_{1}^{-2} e^{\frac{-a_{2}}{C-y_{1}}} e^{\frac{-a_{1}}{y_{1}}} d y_{1}$.

2) General Case of $N$ Subcarriers: Calculating the OP for a larger number of subcarriers using the same technique as for two subcarriers to derive $\operatorname{Pr}\left(\prod_{n=1}^{N} u_{n}-e^{r_{1}} \leq \sum_{n=1}^{N} Y_{n}\right)$ would be challenging, due to several numerical integrations involved. Therefore, for the general case, by using meansquared error curve fitting for different numbers of subcarriers, the Gamma distribution was found to have a good agreement with our simulation results in the model $\sum_{n=1}^{N} Y_{n}$.

Figure 3 confirms that although a distribution fitting test would not provide a high confidence level, $\sum_{n=1}^{N} Y_{n}$ can be reasonably approximated by a Gamma distributed random variable, $\Upsilon_{n}(k, \theta)$, whose shape and scale parameters may be calculated based on the mean value and variance of $\sum_{n=1}^{N} Y_{n}$ as follows:

$$
\theta=\frac{\operatorname{Var}\left[\sum_{n=1}^{N} Y_{n}\right]}{\mathrm{E}\left[\sum_{n=1}^{N} Y_{n}\right]}, \quad \kappa=\frac{\mathrm{E}\left[\sum_{n=1}^{N} Y_{n}\right]}{\theta} .
$$

In order to obtain the required statistics (i.e., $\kappa$ and $\theta$ ), we will calculate the mean and variance of $\sum_{n=1}^{N} Y_{n}$, respectively. Specifically, the mean can be written as

$$
\mathrm{E}\left[\sum_{n=1}^{N} Y_{n}\right]=\sum_{n=1}^{N} \mathrm{E}\left[Y_{n}\right]=\sum_{n=1}^{N}\left(\int_{0}^{s_{n}} y_{n} f_{Y_{n}}\left(y_{n}\right) d y_{n}\right) \text {. }
$$

Subsequently, based on (14), we have

$$
\begin{aligned}
\mathrm{E}\left[\sum_{n=1}^{N} Y_{n}\right] & =\sum_{n=1}^{N}\left(\frac{\sigma^{2} s_{n}}{B_{n}} e^{\frac{\sigma^{2}}{B_{n}}} \int_{0}^{s_{n}} \frac{1}{y_{n}} e^{\frac{-\sigma^{2} s_{n}}{B_{n} y_{n}}} d y_{n}\right) \\
& =\sum_{n=1}^{N}\left(\frac{\sigma^{2} s_{n}}{B_{n}} e^{\frac{\sigma^{2}}{B_{n}}} E_{1}\left(\frac{\sigma^{2}}{B_{n}}\right)\right)
\end{aligned}
$$

where $E_{1}$ represents the exponential integral calculation. Subsequently, we can write the variance as

$$
\begin{aligned}
\operatorname{Var}\left[\sum_{n=1}^{N} Y_{n}\right] & =\sum_{n=1}^{N} \operatorname{Var}\left[Y_{n}\right] \\
& =\sum_{n=1}^{N}\left(\int y_{n}^{2} f\left(y_{n}\right) d y_{n}-\mathrm{E}\left[Y_{n}\right]^{2}\right),
\end{aligned}
$$

where we have $\mathrm{E}\left[Y_{n}\right]=\frac{\sigma^{2} s_{n}}{B_{n}} e^{\frac{\sigma^{2}}{B_{n}}} E_{1}\left(\frac{\sigma^{2}}{B_{n}}\right)$. Consequently, based on (14), we have

$$
\begin{aligned}
& \operatorname{Var}\left[\sum_{n=1}^{N} Y_{n}\right]= \\
& \sum_{n=1}^{N}\left(\left(\frac{\sigma^{2} s_{n}}{B_{n}}\right)^{2}\left(\frac{B_{n}}{\sigma}-e^{\frac{\sigma^{2}}{B_{n}}} E_{1}\left(\frac{\sigma^{2}}{B_{n}}\right)-e^{\frac{2 \sigma^{2}}{B_{n}}} E_{1}^{2}\left(\frac{\sigma^{2}}{B_{n}}\right)\right)\right) .
\end{aligned}
$$

By substituting (24) and (26) into (22), the required statistics of the Gamma distributed random variable are obtained and the OP can be written as

$$
\begin{aligned}
\operatorname{Pr}_{1, N}^{\text {out }} & =\operatorname{Pr}\left(\prod_{n=1}^{N} u_{n}-e^{r_{1}} \leq \Upsilon_{n}(\kappa, \theta)\right) \\
& =1-\operatorname{Pr}\left(\Upsilon_{n}(\kappa, \theta) \leq C_{N}\right),
\end{aligned}
$$




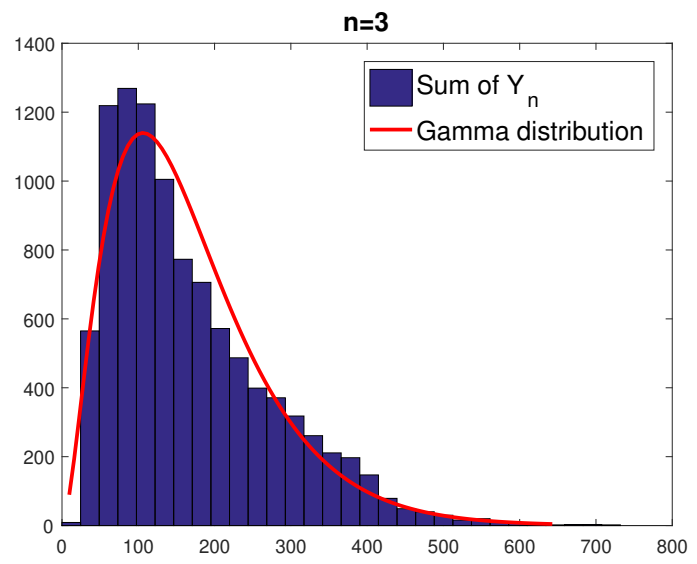

a. Three subcarriers

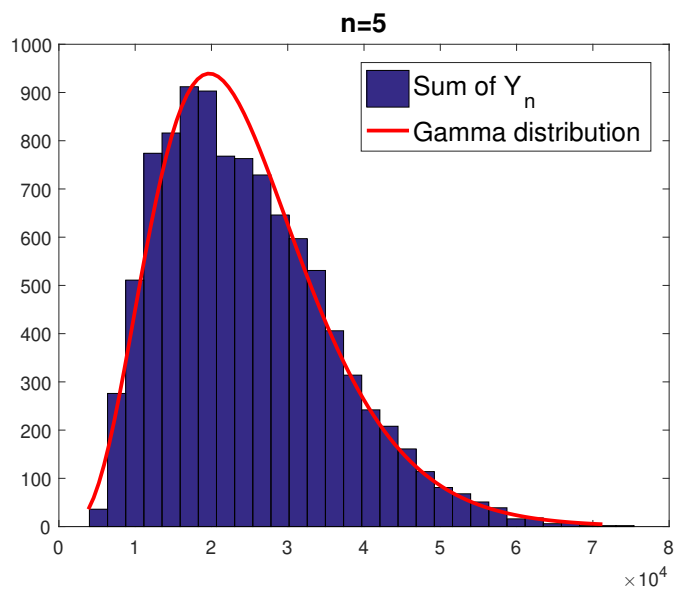

b. Five subcarriers

Fig. 3. The approximation for the sum of $Y_{n}$

where we have $C_{N}=\prod_{n=1}^{N} u_{n}-e^{r_{1}}$. Finally, the OP is calculated as

$$
\begin{aligned}
\operatorname{Pr}_{1, N}^{\text {out }} & =1-F_{\Upsilon_{n}}\left(C_{N}\right) \\
& =1-\frac{1}{\Gamma(\kappa)} \gamma\left(\kappa, \frac{C_{N}}{\theta}\right),
\end{aligned}
$$

where $\gamma\left(\kappa, \frac{C_{N}}{\theta}\right)=\int_{0}^{\frac{C_{n}}{\theta}} t^{\kappa-1} e^{-t} d t$ is the lower incomplete Gamma function.

\section{B. Outage Probability of User 2}

In this subsection, we analyze the OP of user 2 . We define the outage expression of user 2 as

$$
\operatorname{Pr}_{2, N}^{\text {out }}=1-\operatorname{Pr}_{2, N}^{\text {suc }} \times \operatorname{Pr}_{1 \rightarrow 2, N}^{\text {suc }} \text {. }
$$

where $\operatorname{Pr}_{2, N}^{\text {suc }}=\operatorname{Pr}\left(R_{2}>r_{2}\right)$ represents the successful probability of user 2 conditioned on successful SIC of user 1's signal and $\operatorname{Pr}_{1 \rightarrow 2, N}^{\text {suc }}=\operatorname{Pr}\left(R_{1 \rightarrow 2, N}>r_{1 \rightarrow 2, N}\right)$ denotes the probability of the success event of user 2 detecting the message of user 1 . Therefore, in order to calculate the outage expression of user 2, we first need to derive $\operatorname{Pr}_{2, N}^{\text {suc }}$ and $\operatorname{Pr}_{1 \rightarrow 2, N}^{\text {suc }}$.

First, to derive $\operatorname{Pr}_{2, N}^{\text {suc }}$, we represent it as

$$
\begin{aligned}
\operatorname{Pr}_{2, N}^{\mathrm{suc}} & =\operatorname{Pr}\left(\sum_{n=1}^{N} \log \left(1+\frac{\beta_{2, n} h_{2, n}}{\sigma^{2}}\right)>r_{2}\right) \\
& =\operatorname{Pr}\left(\prod_{n=1}^{N}\left(1+\frac{\beta_{2, n} d_{2}^{-\lambda} \chi_{2, n}}{\sigma^{2}}\right)>e^{r_{2}}\right) .
\end{aligned}
$$

where $R_{2}=\sum_{n=1}^{N} \log \left(1+\frac{\beta_{2, n} h_{2, n}}{\sigma^{2}}\right)$ is the achievable rate of user 2, conditioned on the successful SIC of user 1's signal. Let us now define $l_{n}=\frac{\beta_{2, n} d_{2}^{-\lambda}}{\sigma^{2}}$ and $W_{n}=1+l_{n} \chi_{2, n}$, then we have:

$$
\operatorname{Pr}_{2, N}^{\mathrm{suc}}=\operatorname{Pr}\left(\prod_{n=1}^{N} W_{n}>e^{r_{2}}\right) .
$$

In order to calculate $\operatorname{Pr}_{2, N}^{\mathrm{suc}}$, we first determine the probability distribution of $W=\prod_{n=1}^{N} W_{n}$. Since $\chi_{2, n}$ has an exponential distribution and $l_{n}$ has a fixed value, $W_{n}$ is a shifted exponential random variable. According to [39], the PDF of a shifted exponential random variables is defined as $f(x)=\gamma e^{-\gamma(x-\alpha)}$ for $\alpha>0$, where $\gamma$ is a rate parameter and $\alpha$ is a shift parameter.

Since $W_{n}$ is a shifted exponential random variable with $\gamma_{n}=\frac{1}{l_{n}}$ and $\alpha_{n}=1$, the PDF of $W_{n}$ given by $f_{W_{n}}\left(w_{n}\right)=$ $\frac{1}{l_{n}} e^{\frac{1}{l_{n}}\left(w_{n}-1\right)}$. The Mellin transform [40] of $W_{n}$ is defined as

$$
\begin{aligned}
\mathcal{M}_{W_{n}}\left\{f_{W_{n}}\left(w_{n}\right) \mid s\right\} & =\int_{0}^{\infty} w_{n}^{s-1} f_{W_{n}}\left(w_{n}\right) d x_{n} \\
& =e^{1 / l_{n}} l_{n}^{s-1} \Gamma\left(s, 1 / l_{n}\right),
\end{aligned}
$$

where $\Gamma\left(s, 1 / l_{n}\right)=\int_{1 / l_{n}}^{\infty} t^{s-1} e^{-t} d t$ is the upper incomplete Gamma function. Based on the inherent property of the Mellin transform, namely that the Mellin transform of the product of independent random variables can be expressed as the product of the individual Mellin transforms, we arrive at:

$$
\mathcal{M}_{W}\left\{f_{W}(w) \mid s\right\}=\prod_{n=1}^{N} \mathcal{M}_{W_{n}}\left\{f_{W_{n}}\left(w_{n}\right) \mid s\right\} .
$$

Consequently, based on (32), the Mellin transform of $W=$ $\prod_{n=1}^{N} W_{n}$ will be

$$
\begin{aligned}
\mathcal{M}_{W}\left\{f_{W}(w) \mid s\right\} & =\prod_{n=1}^{N}\left(e^{1 / l_{n}} l_{n}^{s-1} \Gamma\left(s, 1 / l_{n}\right)\right) \\
& =K_{N} C_{N}^{s-1} \prod_{n=1}^{N} \Gamma\left(s, 1 / l_{n}\right),
\end{aligned}
$$

where we have $K_{N}=\prod_{n=1}^{N} e^{1 / l_{n}}$ and $C_{N}=\prod_{n=1}^{N} l_{n}$. Therefore, having established the Mellin transform of $W$, the corresponding PDF of $W$ is calculated by integrating $\mathcal{M}_{W}\left\{f_{W}(w) \mid s\right\}$ as

$$
\begin{aligned}
f_{W}(w) & =\frac{1}{2 \pi i} \int_{c-i \infty}^{c+i \infty} \mathcal{M}_{W}\left\{f_{W}(w) \mid s\right\} w^{-s} d s \\
& =\frac{K_{N}}{C_{N}} \mathcal{H}_{0, N}^{N, 0}\left[\frac{w}{C_{N}} \mid \begin{array}{l}
----- \\
\left(0,1,1 / l_{1}\right), \ldots,\left(0,1,1 / l_{N}\right)
\end{array}\right],
\end{aligned}
$$


where $\prod_{n=1}^{N} \alpha_{n}<w<\infty$, and $H_{p, q}^{m, n}[r]$ represents the generalized upper incomplete Fox's H function [41] . This function is defined as

$$
\begin{aligned}
H_{p, q}^{m, n}[r] & \triangleq H_{p, q}^{m, n}\left[r \mid \begin{array}{l}
\left(a_{i}, \alpha_{i}, A_{i}\right)_{1, p} \\
\left(b_{j}, \beta_{j}, B_{j}\right)_{1, q}
\end{array}\right] \\
& \triangleq H_{p, q}^{m, n}\left[\begin{array}{l|l}
\left(a_{1}, \alpha_{1}, A_{1}\right), \ldots,\left(a_{p}, \alpha_{p}, A_{p}\right) \\
\left(b_{1} \beta_{1}, B_{1}\right), \ldots,\left(b_{q} \beta_{q}, B_{q}\right)
\end{array}\right],
\end{aligned}
$$

where $m, n, p$ and $q$ are integers such that $0 \leq m \leq q, 0 \leq$ $n \leq p, a_{i}, b_{j} \in \mathbf{C}$ where $\mathbf{C}$ is the field of complex number, and $\alpha_{i}, A_{i}, \beta_{j}$ and $B_{j} \in \mathbf{R}^{+}(1 \leq i \leq p, 1 \leq j \leq q)$ where $\mathbf{R}^{+}$ is the set of positive real numbers. Fox defined the $\mathrm{H}$-function in his celebrated studies of symmetrical Fourier kernel as the Mellin-Barnes type integral [42] as follows

$$
\mathcal{H}_{p, q}^{m, n}[r]=\frac{1}{2 \pi i} \int_{c-i \infty}^{c+i \infty} \mathcal{M}_{p, q}^{m, n}[s] r^{-s} d s,
$$

where we have:

$$
\begin{aligned}
& \mathcal{M}_{p, q}^{m, n}[s]= \\
& \frac{\prod_{j=1}^{m} \Gamma\left(b_{j}+\beta_{j} s, B_{j}\right) \prod_{i=1}^{n} \Gamma\left(1-a_{i}-\alpha_{i} s, A_{i}\right)}{\prod_{n+1}^{p} \Gamma\left(a_{i}+\alpha_{i} s, A_{i}\right) \prod_{j=m+1}^{q} \Gamma\left(1-b_{j}-\beta_{j} s, B_{j}\right)},
\end{aligned}
$$

while $\mathcal{C}$ is a suitable contour of Barnes type [42] such that the poles of $\Gamma\left(b_{j}+\beta_{j} s, B_{j}\right), j=1, \ldots, m$ lie on the right hand side of the contour and those of $\Gamma\left(1-a_{i}-\alpha_{i} s, A_{i}\right), i=$ $1, \ldots, n$ lie on the left hand side of the contour. Furthermore, the contour $\mathcal{C}$ runs from $c-i \infty$ to $c+i \infty$, so that we have:

(a) $s_{b}=\left(-b_{j}-k\right) / \beta_{j}(j=1, \ldots, m ; k=0,1,2, \ldots)$

(b) $s_{a}=\left(1-a_{i}+k\right) / \alpha_{i}(i=1, \ldots, n ; k=0,1,2, \ldots)$.

Therefore, the value of $\mathcal{C}$ is chosen between $s_{b}$ and $s_{a}$. Our objective is to find the probability of success event for user 2 . According to (31), we have

$$
\operatorname{Pr}_{2, N}^{\mathrm{suc}}=\operatorname{Pr}\left(W>e^{r_{2}}\right)=\int_{e^{r_{2}}}^{\infty} f_{W}(w) d w
$$

Subsequently, based on $f_{W}(w)$ in (35), we have

$\operatorname{Pr}_{2, N}^{\text {suc }}=1-\frac{K_{N}}{C_{N}} \int_{0}^{e^{r_{2}}} \mathcal{H}_{0, N}^{N, 0}\left[\left.\frac{w}{C_{N}}\right|_{\left(0,1,1 / l_{1}\right), \ldots,\left(0,1,1 / l_{N}\right)} ^{-----}\right] d w$.

The difficulty in calculating this probability is in the integration of Fox's $\mathrm{H}$ function, which is relegated to Appendix A. Finally, based on (64), the corresponding probability of success for user 2 may be expressed as:

$$
\begin{aligned}
& \operatorname{Pr}_{2, N}^{\text {suc }}= \\
& 1-K_{N}\left(\mathcal{H}_{N+1,1}^{0, N+1}\left[\frac{e^{r_{2}}}{C_{N}} \mid \begin{array}{l}
(1,1,0) \\
(0,1,0),\left(1,1,1 / l_{1}\right), \ldots,\left(1,1,1 / l_{N}\right)
\end{array}\right]\right) .
\end{aligned}
$$

In addition, to derive the OP of user 2 , it is necessary to calculate the probability of the success event of user 2 detecting the message of user 1 , the corresponding probability is defined as $\operatorname{Pr}_{1 \rightarrow 2, N}^{\text {suc }}=\operatorname{Pr}\left(R_{1 \rightarrow 2}>r_{1 \rightarrow 2}\right)$, where $R_{1 \rightarrow 2}=$ $\sum_{n=1}^{N} \log \left(1+\frac{\beta_{1, n} h_{2, n}}{\sigma^{2}+\beta_{2, n} h_{2, n}}\right)$ represents the achievable rate of detecting user 1's message at user 2 and $r_{1 \rightarrow 2}$ is the target rate. By applying the same analysis method for OP of user 1, the success probability for the case of two subcarriers can be expressed as (42), where $\tilde{B}_{1}=\beta_{2, n} d_{2}^{-\lambda}$ and we have $\tilde{I}(t)=\int_{0}^{t} y_{1}^{-2} e^{\frac{-a_{2}}{\tilde{C}-y_{1}}} e^{\frac{-\tilde{\alpha} y_{1}}{y_{1}}} d y_{1}$. For the case of $N$ subcarriers, this success probability will be $\operatorname{Pr}_{1 \rightarrow 2, N}^{\mathrm{suc}}=\frac{1}{\Gamma(\tilde{k})} \gamma\left(\tilde{k}, \frac{\tilde{C_{n}}}{\tilde{\theta}}\right)$.

\section{OUtage Probability ANALysis for an ARbitrary NUMBER OF USERS}

In this section, we extend the OP analysis for the general case of multiplexing an arbitrary number of users per subcarrier. Let us assume that we have $K$ users in this system and the detection order is based on the distance from the base station to the users, $d_{1}>d_{2}>\cdots>d_{k}>\cdots>d_{K}$. Thus, the total data rate of each user over all the shared subcarriers can be expressed as

$$
\begin{aligned}
& R_{k}= \\
& \begin{cases}\sum_{n=1}^{N} \log \left(1+\frac{\beta_{k, n} h_{k, n}}{\sigma^{2}+\sum_{j=k+1}^{K} \beta_{j, n} h_{k, n}}\right), & k \in\{1,2, . ., K-1\} \\
\sum_{n=1}^{N} \log \left(1+\frac{\beta_{K, n} h_{K, n}}{\sigma^{2}}\right), & k=K .\end{cases}
\end{aligned}
$$

Each subcarrier can be allocated to $K$ users. When relying on a fixed power allocation method, the transmit power allocations of different users will follow $\beta_{1, n}>\beta_{2, n}>\cdots>\beta_{K, n}$. Hence, the outage probability of user 1 (the farthest user) over $N$ subcarriers is defined as

$$
\begin{aligned}
\operatorname{Pr}_{1, N}^{\text {out }} & =\operatorname{Pr}\left(R_{1} \leq r_{1}\right) \\
& =\operatorname{Pr}\left(\sum_{n=1}^{N} \log \left(1+\frac{\beta_{1, n} h_{1, n}}{\sigma^{2}+\sum_{j=2}^{K} \beta_{j, n} h_{1, n}}\right) \leq r_{1}\right) .
\end{aligned}
$$

Let us now reformulate the data rate of user 1 by introducing signal power, Rayleigh fading, and path loss coefficients, yielding:

$$
\begin{aligned}
R_{1} & =\sum_{n=1}^{N} \log \left(1+\frac{\beta_{1, n} h_{1, n}}{\sigma^{2}+\sum_{j=2}^{K} \beta_{j, n} h_{1, n}}\right) \\
& =\sum_{n=1}^{N} \log \left(\frac{\sigma^{2}+\left(\beta_{1, n}+\sum_{j=2}^{K} \beta_{j, n}\right) d_{1}^{-\lambda} \chi_{1, n}}{\sigma^{2}+\sum_{j=2}^{K} \beta_{j, n} d_{1}^{-\lambda} \chi_{1, n}}\right) .
\end{aligned}
$$

Upon defining $A_{n}^{1}=\left(\beta_{1, n}+\sum_{j=2}^{K} \beta_{j, n}\right) d_{1}^{-\lambda}$ and $B_{n}^{1}=$ $\sum_{j=2}^{K} \beta_{j, n} d_{1}^{-\lambda}$, the data rate of user 1 is expressed as

$$
R_{1}=\sum_{n=1}^{N} \log \left(\frac{\sigma^{2}+A_{n}^{1} \chi_{1, n}}{\sigma^{2}+B_{n}^{1} \chi_{1, n}}\right)
$$

where $\chi_{1, n}$ follows an exponential distribution with a mean of one. Comparing the expression of the achievable data rate of user 1 in the case of $K$ users to the case of two users (i.e., (6)), it is clear that it has a similar form, except that the parameters of $A_{n}^{1}$ and $B_{n}^{1}$ hold different values. Therefore, the derivation of the OP expressions of user 1 over two or $N$ subcarriers for the case of $K$ users will follow the same steps (i.e., (7)-(20) $\&$ (21)-(27)) as that of the case of two users.

For user $k$, when $1<k<K$, to analyse the outage probability, it is necessary to calculate the success probability of the event of user $k$ detecting both its own message as well as the message of users who are farther from user $k$. In other words, the corresponding outage probability of user $k$ can be obtained as

$$
\operatorname{Pr}_{k, N}^{\text {out }}=1-\operatorname{Pr}_{k, N}^{\mathrm{suc}} \times \prod_{j=1}^{k-1} \operatorname{Pr}_{j \rightarrow k, N}^{\mathrm{suc}}
$$




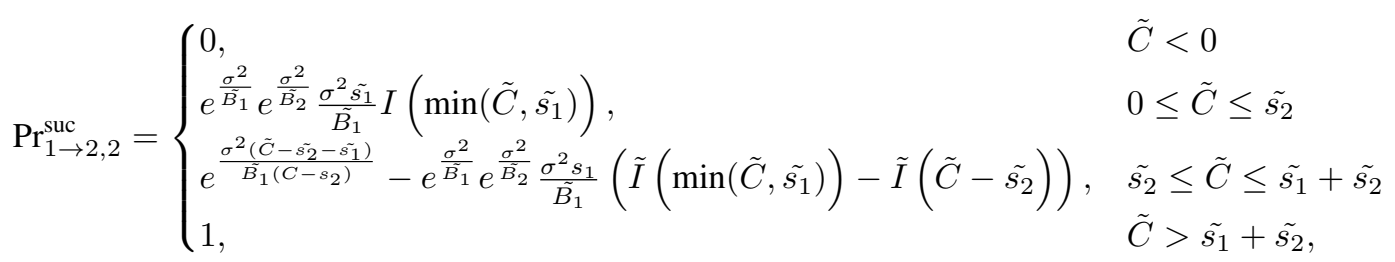

where $\operatorname{Pr}_{k, N}^{\mathrm{suc}}$ is given as

$$
\operatorname{Pr}_{k, N}^{\text {suc }}=\operatorname{Pr}\left(R_{k}>r_{k}\right) \text {. }
$$

The rate of user $k$ can be written as $R_{k}=\sum_{n=1}^{N} \log \left(\frac{\sigma^{2}+A_{n}^{k} \chi_{1, n}}{\sigma^{2}+B_{n}^{k} \chi_{1, n}}\right)$ where $A_{n}^{k}=\left(\beta_{k, n}+\sum_{j=k+1}^{K} \beta_{j, n}\right) d_{k}^{-\lambda}$ and $B_{n}^{k}=$ $\sum_{j=k+1}^{K} \beta_{j, n} d_{k}^{-\lambda}$. Moreover, $\operatorname{Pr}_{j \rightarrow k, N}^{\mathrm{suc}}$ represents the success probability of the event of user $k$ detecting the message of user $j<k$ who is farther away from user $k$ as

$$
\operatorname{Pr}_{j \rightarrow k, N}^{\text {suc }}=\operatorname{Pr}\left(R_{j \rightarrow k}>r_{j \rightarrow k}\right),
$$

where the achievable rate of user $k$ detecting the message of user $j$ is

$$
R_{j \rightarrow k}=\sum_{n=1}^{N} \log \left(1+\frac{\beta_{j, n} h_{k, n}}{\sigma^{2}+\sum_{i=j+1}^{K} \beta_{i, n} h_{k, n}}\right) .
$$

This rate can be rewritten as $R_{j \rightarrow k}=\sum_{n=1}^{N} \log \left(\frac{\sigma^{2}+A_{n}^{j \rightarrow k} \chi_{1, n}}{\sigma^{2}+B_{n}^{j \rightarrow k} \chi_{1, n}}\right)$ where $A_{n}^{j \rightarrow k}=\left(\beta_{j, n}+\sum_{i=j+1}^{K} \beta_{i, n}\right)^{n=1} d_{k}^{-\lambda}$ and $B_{n}^{j \rightarrow k}=$ $\sum_{i=j+1}^{K} \beta_{i, n} d_{k}^{-\lambda}$. Therefore, the same approach as in (7)-(20) \& (21)-(27) can be applied for deriving the closed-form expressions of $\operatorname{Pr}_{j \rightarrow k, N}^{\mathrm{suc}}$ since it follows the same form as in (6).

The outage probability of user $K$ is obtained as

$$
\operatorname{Pr}_{K, N}^{\text {out }}=1-\operatorname{Pr}_{K, N}^{\text {suc }} \times \prod_{j=1}^{K-1} \operatorname{Pr}_{j \rightarrow K, N}^{\text {suc }},
$$

The success probability of user $K$ detecting the messages of other users can be derived by adopting the same method as for the case of user $k$ when $1<k<K$. What is different however, is the analysis of $\operatorname{Pr}_{K, N}^{\text {suc }}$, which is expressed as

$$
\begin{aligned}
\operatorname{Pr}_{K, N}^{\mathrm{suc}} & =\operatorname{Pr}\left(R_{K}>r_{K}\right) \\
& =\operatorname{Pr}\left(\sum_{n=1}^{N} \log \left(1+\frac{\beta_{K, n} d_{K}^{-\lambda} \chi_{K, n}}{\sigma^{2}}\right)>r_{K}\right) \\
& =\operatorname{Pr}\left(\prod_{n=1}^{N}\left(1+\frac{\beta_{K, n} d_{k}^{-\lambda} \chi_{K, n}}{\sigma^{2}}\right)>e^{r_{K}}\right),
\end{aligned}
$$

The achievable rate of user $K$ is given by the sum of the logarithm of shifted exponential random variables. Hence, we can apply the generalized upper incomplete Fox's H function to obtain the success event of user $K$, as seen in steps (29)(40). Here, we have $\hat{l}_{n}=\frac{\beta_{K, n} d_{K}^{-\lambda}}{\sigma^{2}}, \hat{K}_{N}=\prod_{n=1}^{N} e^{1 / \hat{l}_{n}}$ and $\hat{C}_{N}=\prod_{n=1}^{N} \hat{l}_{n}$. Therefore,

$$
\begin{aligned}
& \operatorname{Pr}_{K, N}^{\mathrm{suc}}=\operatorname{Pr}\left(R_{K}>r_{K}\right) \\
& =1-\hat{K}_{N}\left(\mathcal{H}_{N+1,1}^{0, N+1}\left[\frac{e^{r_{k}}}{\hat{C}_{N}} \mid \begin{array}{l}
(1,1,0) \\
(0,1,0),\left(1,1,1 / \hat{l}_{1}\right), \ldots,\left(1,1,1 / \hat{l}_{N}\right)
\end{array}\right]\right) .
\end{aligned}
$$

\section{Simulation RESUlTS}

We present several simulation results for the OP for the case of two users and three users. In the simulation results, generally, the SNR of User $K$ (the user closest to the BS) is treated as a unified benchmark $\operatorname{SNR}_{K, n}=\frac{\beta_{K, n} d_{K}^{-\lambda}}{\sigma^{2}}$. We assume that both the distance and power changes follow the same scale, i.e., the distance ratio variable is defined as $\rho=$ $\frac{d_{1}}{d_{2}}=\cdots=\frac{d_{K-1}}{d_{K}}$, and the power ratio coefficient of $t=$ $\frac{\beta_{1, n}}{\beta_{2, n}}=\cdots=\frac{\beta_{K-1, n}}{\beta_{K, n}}$. Therefore, the average received SNR of user $k$ can be formulated as

$$
\begin{aligned}
\mathrm{SNR}_{k, n} & =\frac{\beta_{k, n} d_{k}^{-\lambda}}{\sigma^{2}} \\
& =t^{K-k} \times\left(\rho^{K-k}\right)^{-\lambda} \times \mathrm{SNR}_{K, n}, \quad k=1, \ldots K-1
\end{aligned}
$$

For the case of two users, we have provided numerical results for both users to validate the accuracy of the expressions derived. The corresponding data rate of user 2 can be written as $R_{2}=\sum_{n=1}^{N} \log \left(1+\chi_{2, n} \mathrm{SNR}_{2, n}\right)$. In order to guarantee that SIC is successful, more transmission power is needed to the far user that experiences the worst-case channel condition [43]. Hence, we only explore the power ratio coefficients which are larger than one, i.e., $t=\frac{\beta_{1, n}}{\beta_{2, n}}>1$.

Hence, the data rate for user 1 can be written as

$$
\begin{aligned}
R_{1} & =\sum_{n=1}^{N} \log \left(\frac{\sigma^{2}+\left(\beta_{1, n}+\beta_{2, n}\right) d_{1}^{-\lambda} \chi_{1, n}}{\sigma^{2}+\beta_{2, n} d_{1}^{-\lambda} \chi_{1, n}}\right) \\
& =\sum_{n=1}^{N} \log \left(\frac{\sigma^{2}+(t+1) \mathrm{SNR}_{2, n} \sigma^{2} \rho^{-\lambda} \chi_{1, n}}{\sigma^{2}+\mathrm{SNR}_{2, n} \sigma^{2} \rho^{-\lambda} \chi_{1, n}}\right)
\end{aligned}
$$

where the corresponding average received SNR of user 1 at subcarrier $n$ is defined as $\mathrm{SNR}_{1, n}=\frac{\beta_{1, n} d_{1}^{-\lambda}}{\sigma^{2}}=t \times \rho^{-\lambda} \times$ $\mathrm{SNR}_{2, n}$. As expected, when $\rho$ increases, the data rate of user 1 decreases and the corresponding OP increases. Without loss of generality, in our simulations, we assume that each user has the same power level over the different subcarriers. In the following, we analyze the OP of user 1 and user 2, respectively. 


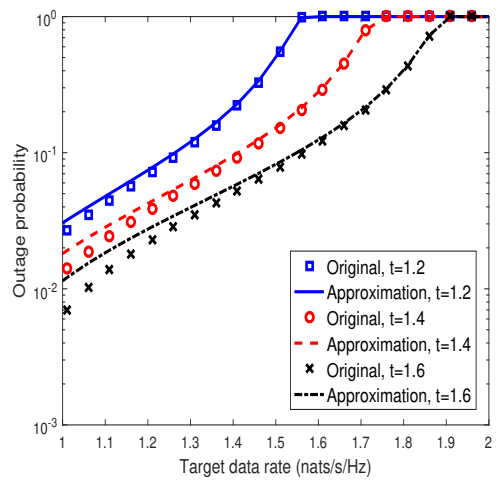

(a) Two subcarriers

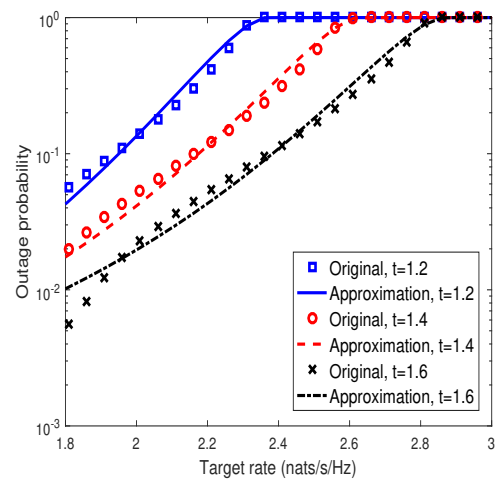

(b) Three subcarriers

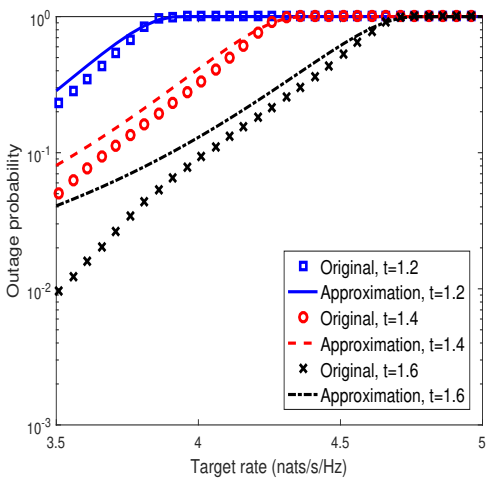

(c) Five subcarriers

Fig. 4. The outage probability of user 1 vs target rate, with different power ratio coefficients $t\left(\mathrm{SNR}_{2, n}=18 d B, \rho=1.5\right)$

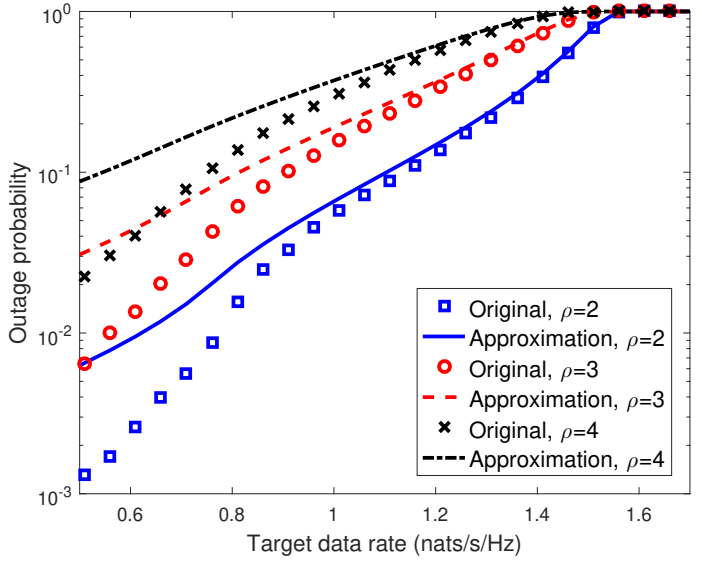

(a) $\mathrm{SNR}_{2, n}=18 d B$

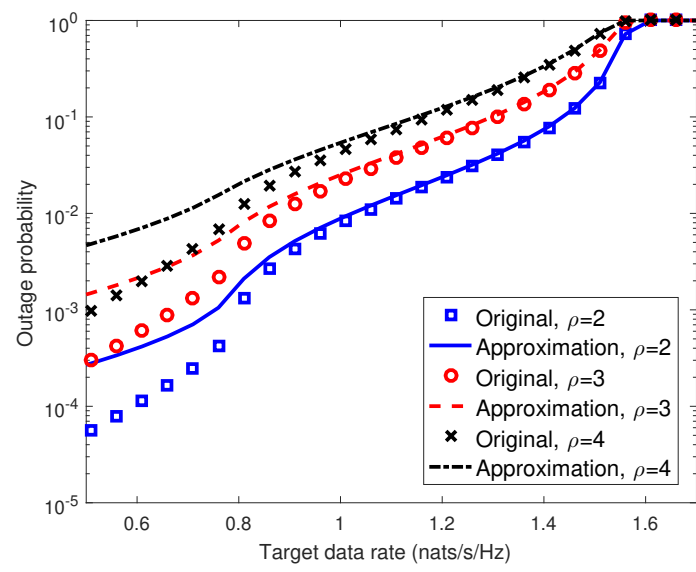

(b) $\mathrm{SNR}_{2, n}=25 d B$

Fig. 5. The outage probability of user 1 vs target rate, with different distance ratios $\rho(t=1.2)$

\section{A. User 1}

Figure 4 depicts the relationship between the target data rate and the OP for three cases having different numbers of subcarriers. The value of $\mathrm{SNR}_{2, n}$ is fixed to $18 d B$. It is observed that the OP of user 1 increases with the target rate and decreases with the power ratio coefficient $t$. This observation implies that NOMA systems achieve a good performance when the power levels of the two users are rather different. This is helpful, when determining the optimal power allocation policy considering a specific user distribution. The approximation matches closely to the true ones, however, the gap between the approximated and original OP increases with $t$ for all three cases, particularly in the lower target rate region. Likewise, as the number of subcarriers increases, the accuracy of the approximation decreases. This confirms accuracy of the OP analysis for the case of two subcarriers. In Figure 5, we focus our attention on the effects of the distance ratio $\rho$ on the OP of two subcarriers. As the target rate and $\rho$ increase, the OP of user 1 increases. Obviously, the accuracy of the approximated OPs is similar for different values of $\rho$. As the value of $\mathrm{SNR}_{2, n}$ increases, the gap between the approximated and original OPs shrinks. The OP approximation error versus
$\mathrm{SNR}_{2, n}$ is demonstrated in Figure 6. The OP approximation error is defined as the difference between the original OP based on numerical results and the approximated OP based on the analytical results.

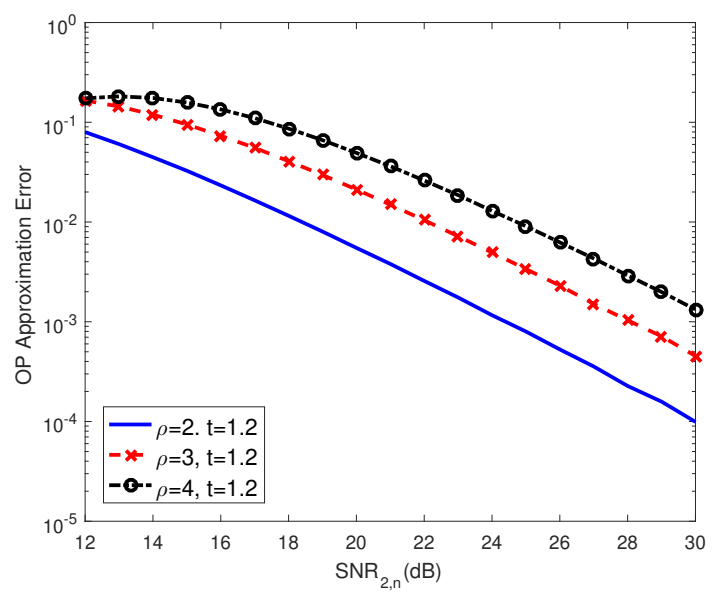

Fig. 6. OP approximation error versus $\mathrm{SNR}_{2, n}(r=0.8)$ 


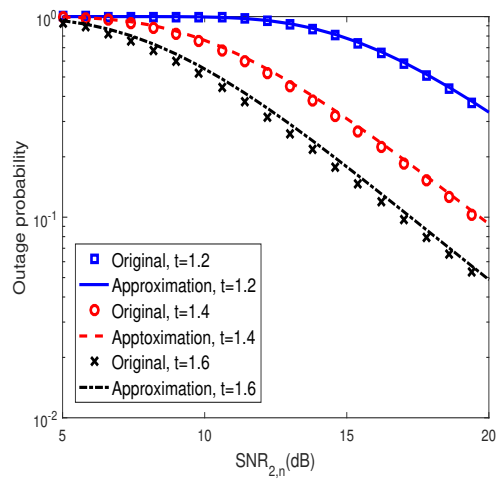

(a) Two subcarriers $(r=1.5)$

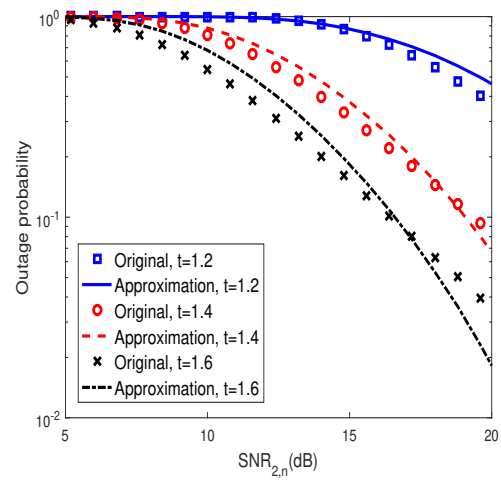

(b) Three subcarriers $(r=2.25)$

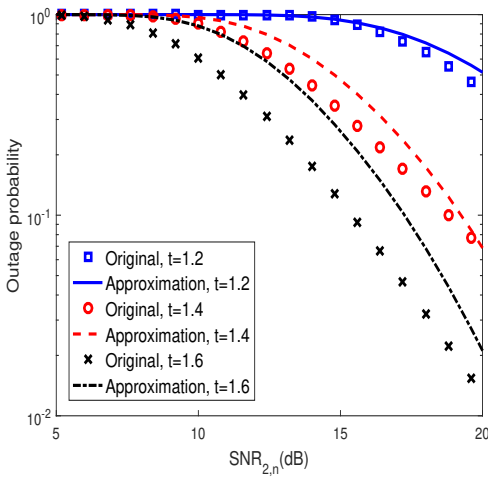

(c) Five subcarriers $(r=3.75)$

Fig. 7. The outage probability of user 1 vs $\mathrm{SNR}_{2, n}$, with different power ratio coefficients $t(\rho=1.5)$

Figure 7 and Figure 8 illustrate the effects of $\mathrm{SNR}_{2, n}$ on the OP of user 1 for different number of subcarriers. As the value of $\mathrm{SNR}_{2, n}$ increases, the OP decreases. These two figures analyze the impact of various power ratios $t$ and distance ratios $\rho$ on the OP considering different numbers of subcarriers. The OP decreases with the power ratio and increases with the distance ratio. These figures highlight the necessity of carefully choosing $t$ taking into account the SNR (implying the total available power budget) and $\rho$ (implying the user distributions). For instance, at $\mathrm{SNR}_{2, n}=20 \mathrm{~dB}$ and $\rho=1.5$, opting for $t=1.6$ reduces the OP by a factor of 25 over that of $t=1.2$. Moreover, the difference between the original and the approximated OP becomes larger for high values of power ratio and distance ratio. The accuracy of the approximation is reduced upon increasing the number of subcarriers.

\section{B. User 2}

In this subsection, we verify the accuracy of the probability of success expression derived in (41) for the second user. This is defined as $\operatorname{Pr}_{2}^{\text {suc }}=\operatorname{Pr}\left(\sum_{n=1}^{N} \log \left(1+\frac{\beta_{2, n} h_{2, n}}{\sigma^{2}}\right) \geq r_{2}\right)$, i.e., the probability of satisfying the total rate of an individual user over all subcarriers, which is calculated by using the generalized upper incomplete Fox's $\mathrm{H}$ function. It is shown that the simulation results closely match the analytical results for all three cases.

Figure 9 shows the relationship between the probability of success event and the target rate. The value of average received SNR for user 2 on subcarrier $n$ is set to $18 \mathrm{~dB}$. As the target data rate increases, the probability of success event decreases. By contrast, the higher the number of subcarriers, the higher the success rate is. In Figure 10, we observe that as $\mathrm{SNR}_{2, n}$ increases, the probability of success also increases. Additionally, the success rate of a larger number of subcarriers is higher than that of a lower number of subcarriers $N$. This figure demonstrates that the derived probability of success for user 2 in terms of detecting its own message (i.e. $\mathrm{Pr}_{2, N}^{\text {suc }}$ ) matches perfectively to the simulation results.

Let us now analyze the OP of user 2 and compare this to that of user 1 . For the sake of simplicity, we assume an identical number of subcarriers for both users. Observe from Figure
11 , that as expected, the $\mathrm{OP}$ of both users increases with the target rate. The OP of user 1 is always higher than that of user 2 since user 1 suffers from more interference than user 2. The diversity gain of having higher degrees of freedom in the case of five subcarriers is clear from the lower OP that is achievable for a fixed target rate. The diversity order will be further discussed in Figure 13.

\section{Comparison with OMA}

For an OMA system, each subcarrier is used exclusively by a single user only. If $\mathcal{N}_{k}$ denotes the set of subcarriers allocated to user $k$, its rate is given by

$$
R_{k}^{\mathrm{OMA}}=\sum_{n \in \mathcal{N}_{k}} \log \left(1+\frac{\beta_{k, n} h_{k, n}}{\sigma^{2}}\right),
$$

Hence, the corresponding outage probability is

$$
\begin{aligned}
& \operatorname{Pr}\left(R_{k}^{\mathrm{OMA}} \leq r_{k}\right) \\
& =\frac{K_{\mathcal{N}_{k}}}{C_{\mathcal{N}_{k}}} \int_{0}^{e^{r_{k}}} \mathcal{H}_{0, \mathcal{N}_{k}}^{\mathcal{N}_{k}, 0}\left[\left.\frac{w}{C_{\mathcal{N}_{k}}}\right|_{\left(0,1,1 / l_{1}\right), \ldots,\left(0,1,1 / l_{\mathcal{N}_{k}}\right)} ^{-----}\right] d w,
\end{aligned}
$$

In the following simulations, we compare the OMA and NOMA systems for the case of two users and two subcarriers as well as for the case of three users and three subcarriers. It is observed that the outage performance for each user of NOMA system is superior to that of the OMA system for both cases. This is because the diversity order of the NOMA system is higher than that of the OMA system. It should be noted however that the outage performance of NOMA is sensitive to the values of power ratio $t$ and distance ratio $\rho$.

Moreover, we investigate the diversity gain (which is defined as $\lim _{S N R \rightarrow \infty} \frac{\log \left(\mathrm{Pr}_{\text {out }}\right)}{\log (S N R)}$, where SNR is the ratio of the total transmit power and the receiver noise power) of MC-NOMA for various cases and compare it with that of the OMA scheme. Fig 13 shows that MC-NOMA having two subcarriers achieves a diversity order of approximately two while the diversity order of OMA is one. Furthermore, it can be observed that the diversity order of MC-NOMA associated with $N=3$ is close to three, even though two users share the three subcarriers. 


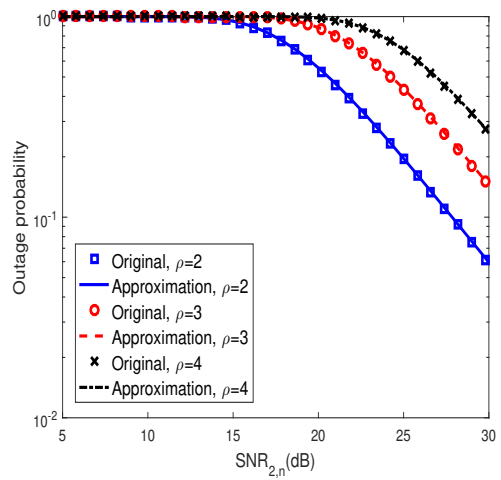

(a) Two subcarriers $(r=1.5)$

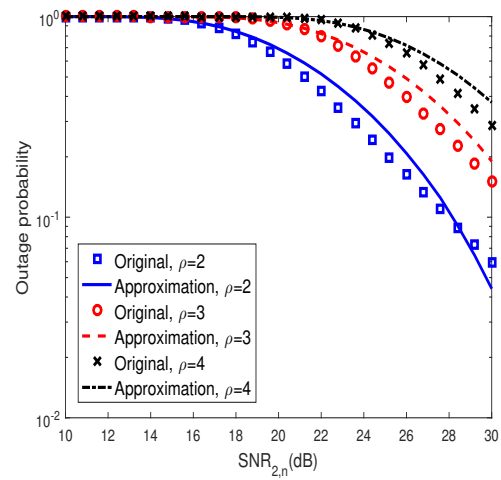

(b) Three subcarriers $(r=2.25)$

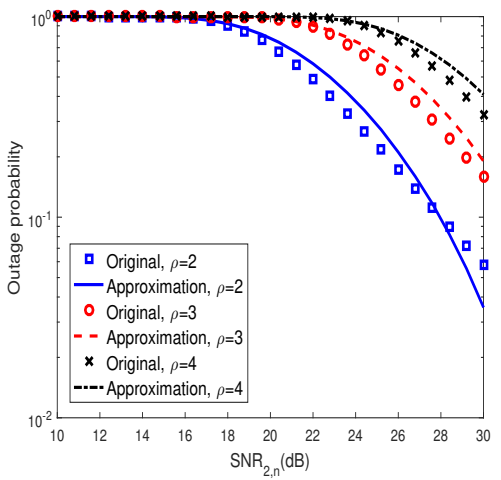

(c) Five subcarriers $(r=3.75)$

Fig. 8. The outage probability of user $1 \mathrm{vs} \mathrm{SNR}_{2, n}$, with different distance ratios $\rho(t=1.2)$

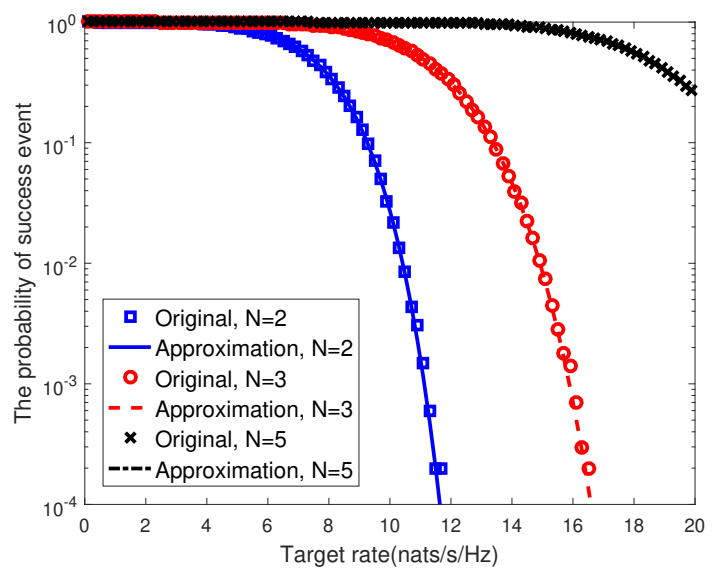

Fig. 9. Success probability of user 2 vs target rate

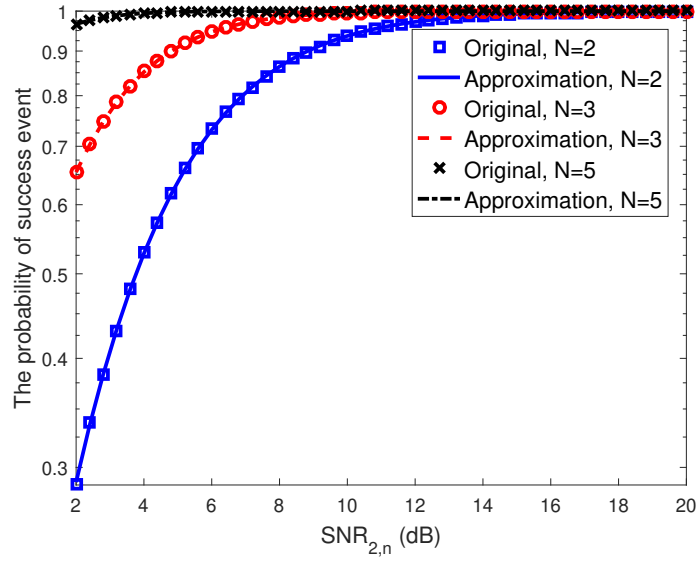

Fig. 10. Success probability of user 2 vs $\mathrm{SNR}_{2, n}$

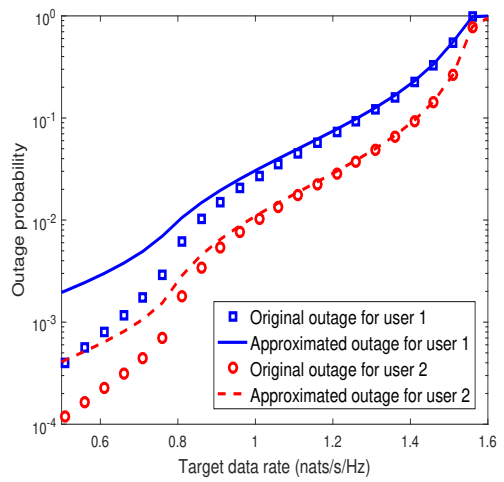

(a) Two subcarriers

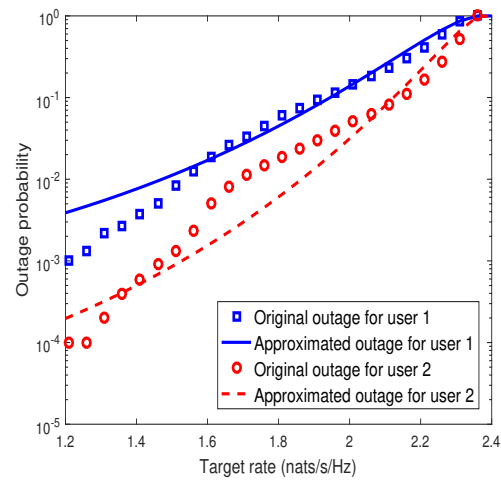

(b) Three subcarriers

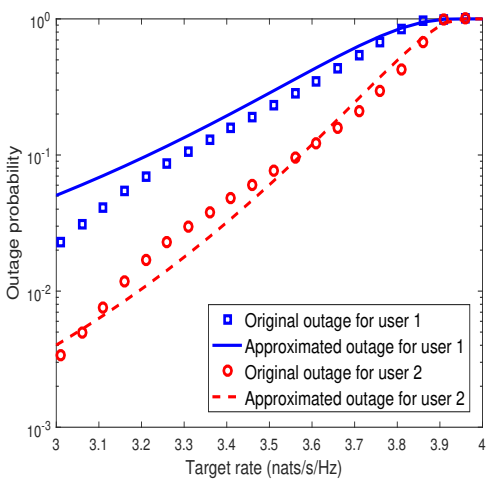

(c) Five subcarriers

Fig. 11. Outage probability of both users vs target rate $\left(\mathrm{SNR}_{2, n}=18 d B, t=1.2, \rho=1.5\right)$

\section{D. $O P$ analysis for $K=3$}

Fig. 14 compares the derived OP against data rate with simulation results for the case of $K=3$ users in each subcarrier. Clearly, the analytical results closely match the simulation results. Compared to Fig 11 (a), this figure shows that the OP for the case of three users sharing each subcarrier is slightly higher than that of two users allocated to each subcarrier.

\section{E. Joint Outage Probability}

Based on the separate outage probabilities, the joint outage probability can be calculated as

$$
\operatorname{Pr}_{\text {out }}^{\text {joint }}=\operatorname{Pr}\left(R_{1}+R_{2} \leq r\right)=\operatorname{Pr}\left(R_{1} \leq r-R_{2}\right)
$$




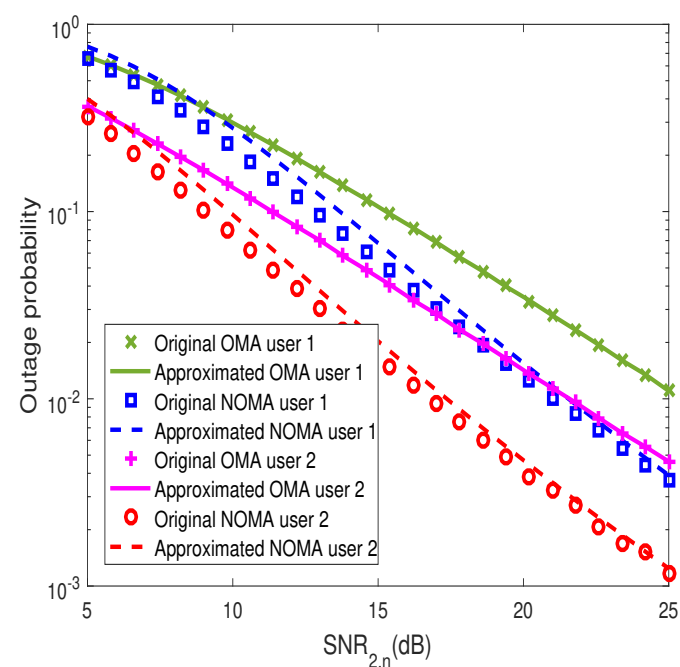

(a) Two users and two subcarriers

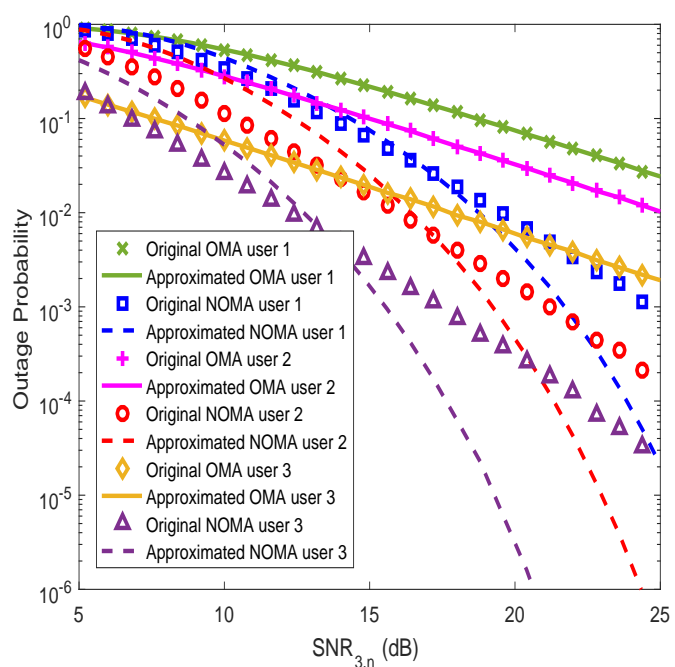

(b) Three users and three subcarriers

Fig. 12. Outage probability comparison between OMA and NOMA systems $(r=1, t=1.2, \rho=1.5)$

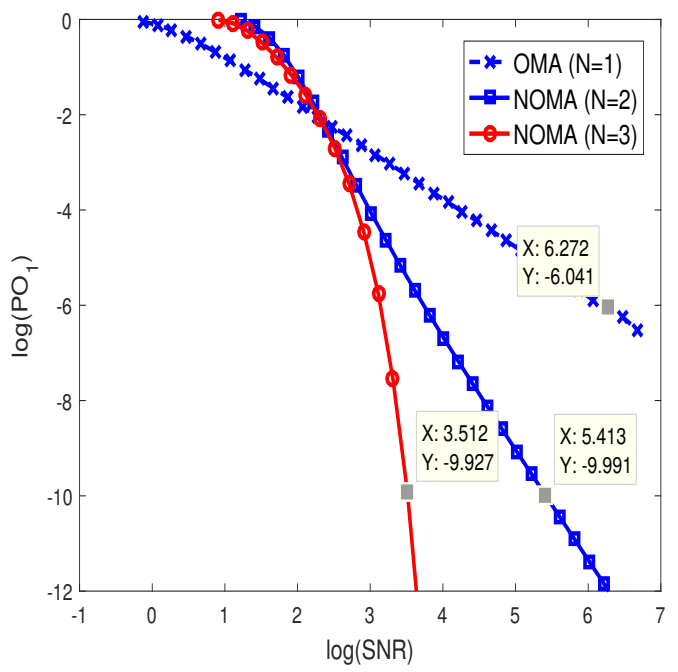

Fig. 13. $\log \left(\mathrm{Pr}_{\text {out }}\right)$ versus $\log (\mathrm{SNR})(r=1, t=1.2, \rho=1.5)$

$$
=\int_{0}^{r} \operatorname{Pr}_{1}^{\text {out }}\left(r-R_{2}\right) f_{R_{2}}\left(R_{2}\right) d R_{2}
$$

where $f_{R_{2}}\left(R_{2}\right)$ is the corresponding PDF of $R_{2}=$ $\sum_{n=1}^{N} \log \left(1+\frac{\beta_{2, n} d_{2}^{-\lambda} \chi_{2, n}}{\sigma^{2}}\right)$. There is no simple or direct way of deriving the PDF of the sum of the logarithm of shifted exponential random variables, although the pdf of the product of shifted exponential random variables is known. Thus, we present $R_{2}$ as $R_{2}=\log \left[\prod_{n=1}^{N}\left(1+\frac{\beta_{2, n} d_{2}^{-\lambda} \chi_{2, n}}{\sigma^{2}}\right)\right]$. Upon assuming $W=\prod_{n=1}^{N} W_{n}$ and $W_{n}=1+\frac{\beta_{2, n} d_{2}^{-\lambda} \chi_{2, n}}{\sigma^{2}}$, we have $R_{2}=\log W$. By changing the variables, we transform the integral (55) as

$$
\operatorname{Pr}_{\text {out }}^{\text {joint }}=\int_{-\infty}^{e^{r}} \frac{1}{W} \operatorname{Pr}_{1}^{\text {out }}(r-\log W) f_{W}(W) d W,
$$

where $f_{W}(W)$ represents the PDF of $W$.

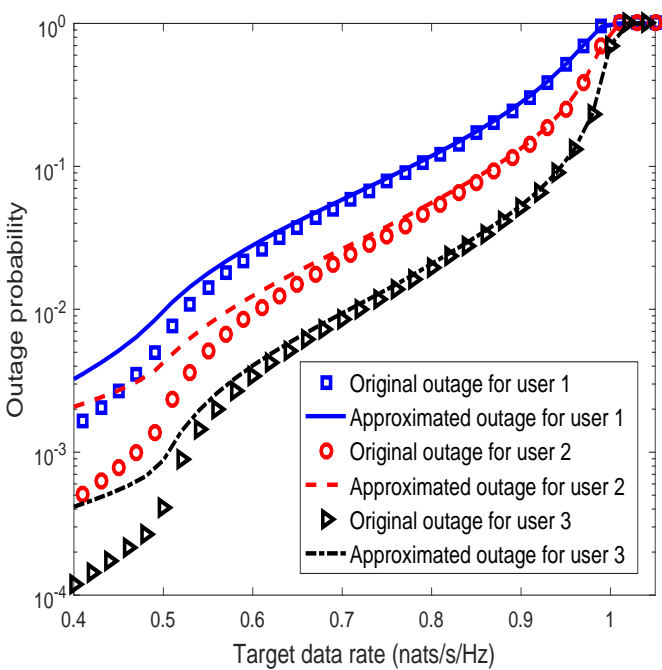

Fig. 14. Outage probability of each user versus target rate $\left(\mathrm{SNR}_{2, n}=\right.$ $18 d B, t=1.2, \rho=1.5)$

The outage probability of user 1 is obtained as $\operatorname{Pr}_{1, N}^{\text {out }}(r-$ $\log W)=1-\frac{1}{\Gamma(\kappa)} \gamma\left(\kappa, \frac{\hat{\mathcal{C}}_{N}}{\theta}\right)$ for the case of $N$ subcarriers according to the steps ranging from (4) to (27), assuming $\hat{\mathcal{C}}_{N}=\prod_{n=1}^{N} u_{n}-e^{r-\log W}$. Moreover, the PDF of $W$ is obtained following the steps (29)-(34) as $f_{W}(W)=$ $\frac{K_{N}}{C_{N}} \mathcal{H}_{0, N}^{N, 0}\left[\frac{w}{C_{N}} \mid \begin{array}{l}----- \\ \left(0,1,1 / l_{1}\right), \ldots,\left(0,1,1 / l_{N}\right)\end{array}\right]$.

Therefore, the joint outage probability will be

$$
\begin{aligned}
& \operatorname{Pr}_{\text {out }}^{\text {joint }}=\int_{-\infty}^{e^{r}} \frac{1}{W}\left(1-\frac{1}{\Gamma(\kappa)} \gamma\left(\kappa, \frac{\hat{\mathcal{C}}_{N}}{\theta}\right)\right) \times \\
& \frac{K_{N}}{C_{N}} \mathcal{H}_{0, N}^{N, 0}\left[\frac{w}{C_{N}} \mid \begin{array}{l}
\left(0,1,1 / l_{1}\right), \ldots,\left(0,1,1 / l_{N}\right) \\
(---
\end{array}\right] d W
\end{aligned}
$$

where we have $K_{N}=\prod_{n=1}^{N} e^{1 / l_{n}}, C_{N}=\prod_{n=1}^{N} l_{n}$ and $l_{n}=\frac{\beta_{2, n} d_{2}^{-\lambda}}{\sigma^{2}}$. This integral can be numerically solved. Here, 
we provide a set of simulation results to examine the joint $\mathrm{OP}$ performance of MC-NOMA system. Fig 15 depicts the joint OP for different numbers of users and subcarriers. Apparently, increasing the number of subcarriers significantly decreases the outage probability as a benefit of increasing the diversity gain achieved by a higher number of subcarriers. The diversity order has already been discussed in Figure 13.

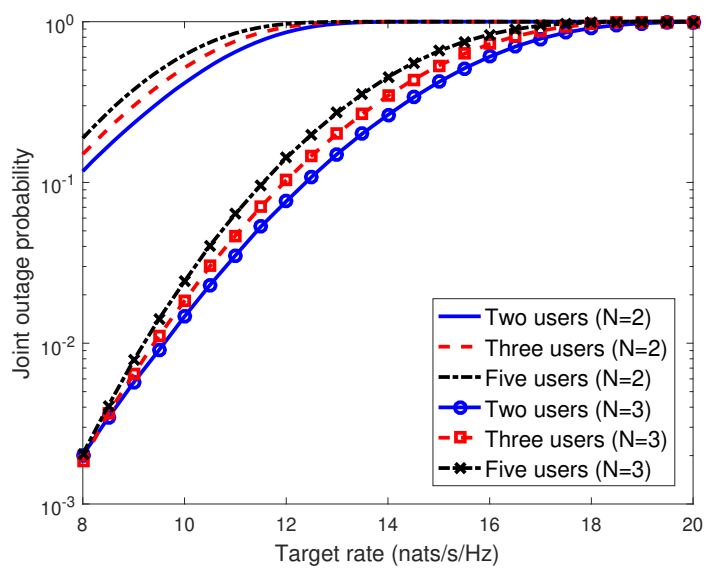

Fig. 15. Joint outage probability versus target rate $\left(\mathrm{SNR}_{2, n}=18 d B, t=1.2\right.$, $\rho=1.5)$

\section{CONCLUSIONS}

In this paper, tractable OP expressions have been derived for the single cell multi-carrier NOMA downlink only relying on the statistical CSI based on approximation techniques. The PDF and CDF of the inverse shifted exponential random variables were formulated for the far user and the OP was derived for the case of two subcarriers. To obtain the OP of far user for the general case of an arbitrary number of subcarriers, the sum of the inverse shifted exponential random variables was approximated by a Gamma distribution. On the other hand, the PDF of the product of shifted exponential random variables was derived for the near user using the Mellin transform and the generalized upper incomplete Fox's $\mathrm{H}$ function. Our analysis and simulation results demonstrate a good accuracy for the approximations.

\section{APPENDIX A \\ THE INTEGRAL OF FOX'S H FUNCTION}

Here, a solution for the integral of Fox's $\mathrm{H}$ function in (40) is formulated as:

$$
\begin{aligned}
& \int_{0}^{r} \mathcal{H}_{p, q}^{m, n}\left[u \mid \begin{array}{c}
\left(a_{i}, \alpha_{i}, A_{i}\right)_{1, p} \\
\left(b_{j}, \beta_{j}, B_{i}\right)_{1, q}
\end{array}\right] d u \\
& =\int_{0}^{r} \frac{1}{2 \pi i} \int_{c-i \infty}^{c+i \infty} \mathcal{M}_{p, q}^{m, n}[s] u^{-s} d s d u \\
& =\frac{1}{2 \pi i} \int_{c-i \infty}^{c+i \infty} \mathcal{M}_{p, q}^{m, n}[s] \quad \int_{0}^{r} u^{-s} d u \quad d s \\
& =\left.\frac{1}{2 \pi i} \int_{c-i \infty}^{c+i \infty} \mathcal{M}_{p, q}^{m, n}[s] \frac{u^{1-s}}{1-s}\right|_{0} ^{r} d s
\end{aligned}
$$

$=\frac{1}{2 \pi i} \int_{c-i \infty}^{c+i \infty} \mathcal{M}_{p, q}^{m, n}[s] \frac{r^{1-s}}{1-s} d s-\frac{1}{2 \pi i} \int_{c-i \infty}^{c+i \infty} \mathcal{M}_{p, q}^{m, n}[s] \frac{0^{1-s}}{1-s} d s ;$

The integral of the $\mathrm{H}$ function depends on the value of $s$. Hence we analyze this for three different cases of $s$.

(a) $s>1$;

$$
\begin{aligned}
& \int_{0}^{r} \mathcal{H}_{p, q}^{m, n}\left[u \mid \begin{array}{c}
\left(a_{i}, \alpha_{i}, A_{i}\right)_{1, p} \\
\left(b_{j}, \beta_{j}, B_{i}\right)_{1, q}
\end{array}\right] d u \\
& =\frac{1}{2 \pi i} \int_{c-i \infty}^{c+i \infty} \mathcal{M}_{p, q}^{m, n}[s] \frac{r^{1-s}}{1-s} d s+\infty ;
\end{aligned}
$$

(b) $s=1$;

$$
\int_{0}^{r} \mathcal{H}_{p, q}^{m, n}\left[u \mid \begin{array}{l}
\left(a_{i}, \alpha_{i}, A_{i}\right)_{1, p} \\
\left(b_{j}, \beta_{j}, B_{i}\right)_{1, q}
\end{array}\right] d u=0
$$

(c) $s<1$;

$$
\begin{aligned}
& \int_{0}^{r} \mathcal{H}_{p, q}^{m, n}\left[u \mid \begin{array}{l}
\left(a_{i}, \alpha_{i}, A_{i}\right)_{1, p} \\
\left(b_{j}, \beta_{j}, B_{i}\right)_{1, q}
\end{array}\right] d u \\
& =\frac{1}{2 \pi i} \int_{c-i \infty}^{c+i \infty} \mathcal{M}_{p, q}^{m, n}[s] \frac{r^{1-s}}{1-s} d s ;
\end{aligned}
$$

According to the definition and properties of the incomplete Gamma function, we obtain the recurrence relations through integration by parts

$$
\begin{aligned}
\Gamma(2-s, 0) & =\int_{0}^{\infty} t^{1-s} e^{-t} d t \\
& =0+\int_{0}^{\infty}(1-s) t^{-s} e^{-t} d t=(1-s) \Gamma(1-s, 0) ;
\end{aligned}
$$

Hence, we have

$$
\begin{aligned}
& \frac{1}{2 \pi i} \int_{c-i \infty}^{c+i \infty} \mathcal{M}_{p, q}^{m, n}[s] \frac{r^{1-s}}{1-s} d s \\
& =r \frac{1}{2 \pi i} \int_{c-i \infty}^{c+i \infty} \mathcal{M}_{p, q}^{m, n}[s] \frac{\Gamma(1-s, 0)}{\Gamma(2-s, 0)} r^{-s} d s \\
& =r \frac{1}{2 \pi i} \int_{c-i \infty}^{c+i \infty} \mathcal{M}_{p+1, q+1}^{m, n+1}[s] r^{-s} d s \\
& =r \mathcal{H}_{p+1, q+1}^{m, n+1}\left[r \mid \begin{array}{l}
\left(a_{i}, \alpha_{i}, A_{i}\right)_{1, p},(0,1,0) \\
\left(b_{j}, \beta_{j}, B_{i}\right)_{1, q},(-1,1,0)
\end{array}\right]
\end{aligned}
$$

By exploiting basic properties of $\mathrm{H}$ functions as $r^{k} \mathcal{H}_{p, q}^{m, n}\left[r \mid \begin{array}{l}\left(a_{i}, \alpha_{i}, A_{i}\right)_{1, p} \\ \left(b_{j}, \beta_{j}, B_{i}\right)_{1, q}\end{array}\right]=\mathcal{H}_{p, q}^{m, n}\left[r \mid \begin{array}{l}\left(a_{i}+k \alpha_{i}, \alpha_{i}, A_{i}\right)_{1, p} \\ \left(b_{j}+k \lambda_{j}, \lambda_{j}, B_{i}\right)_{1, q}\end{array}\right]$, the last integral can be written as

$$
\begin{aligned}
& \frac{1}{2 \pi i} \int_{c-i \infty}^{c+i \infty} \mathcal{M}_{p, q}^{m, n}[s] \frac{r^{1-s}}{1-s} d s \\
& =\mathcal{H}_{p+1, q+1}^{m, n+1}\left[r \mid \begin{array}{l}
\left(a_{i}+\alpha_{i}, \alpha_{i}, A_{i}\right)_{1, p},(1,1,0) \\
\left(b_{j}+\beta_{j}, \beta_{j}, B_{i}\right)_{1, q},(0,1,0)
\end{array}\right] ;
\end{aligned}
$$

In our case, the value of $s$ is always less than the one based on the value of $b_{j}=0$ and $\lambda_{j}=1$. Hence, the integral of the generalized upper incomplete Fox's $\mathrm{H}$ function becomes:

$$
\begin{aligned}
& \int_{0}^{e^{r_{2}}} \mathcal{H}_{0, N}^{N, 0}\left[\left.\frac{w}{C_{N}}\right|_{\left(0,1,1 / l_{1}\right), \ldots,\left(0,1,1 / l_{N}\right)} ^{----}\right] d w \\
& =e^{r_{2}} \mathcal{H}_{N+1,1}^{0, N+1}\left[\frac{e^{r_{2}}}{C_{N}} \mid \begin{array}{l}
(0,1,0) \\
(-1,1,0),\left(0,1,1 / l_{1}\right), \ldots,\left(0,1,1 / l_{N}\right)
\end{array}\right] \\
& =C_{N} \mathcal{H}_{N+1,1}^{0, N+1}\left[\frac{e^{r_{2}}}{C_{N}} \mid \begin{array}{l}
(1,1,0) \\
(0,1,0),\left(1,1,1 / l_{1}\right), \ldots,\left(1,1,1 / l_{N}\right)
\end{array}\right] ;
\end{aligned}
$$




\section{APPENDIX B}

\section{MATHEMATICA CODE FOR GENERALIZED UPPER INCOMPLETE FOX'S H FUNCTION}

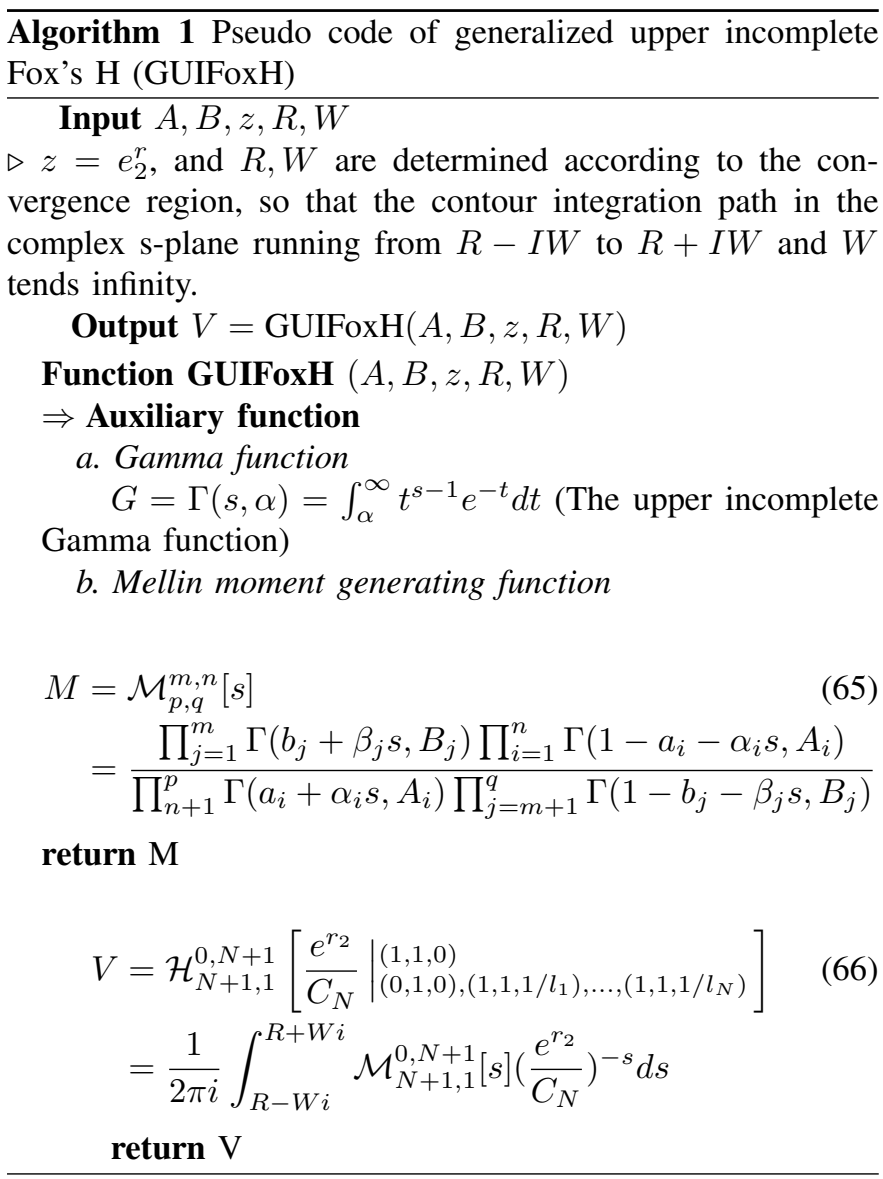

\section{REFERENCES}

[1] Z. Ding, X. Lei, G. K. Karagiannidis, R. Schober, J. Yuan, and V. K. Bhargava, "A survey on non-orthogonal multiple access for $5 \mathrm{G}$ networks: Research challenges and future trends," IEEE Journal on Selected Areas in Communications, vol. 35, no. 10, pp. 2181-2195, Oct. 2017.

[2] L. Dai, B. Wang, Z. Ding, Z. Wang, S. Chen, and L. Hanzo, "A survey of non-orthogonal multiple access for 5G," IEEE Communications Surveys Tutorials, vol. 20, no. 3, pp. 2294-2323, thirdquarter 2018.

[3] Y. Saito, Y. Kishiyama, A. Benjebbour, T. Nakamura, A. Li, and K. Higuchi, "Non-orthogonal multiple access (NOMA) for cellular future radio access," in Vehicular Technology Conference (VTC Spring), Dresden, 2013, pp. 1-5.

[4] Z. Ding, Y. Liu, J. Choi, Q. Sun, M. Elkashlan, C. I, and H. V. Poor, "Application of non-orthogonal multiple access in LTE and 5G networks," IEEE Communications Magazine, vol. 55, no. 2, pp. 185191, Feb. 2017.

[5] L. Dai, B. Wang, Y. Yuan, S. Han, I. Chih-Lin, and Z. Wang, "Nonorthogonal multiple access for 5G: solutions, challenges, opportunities, and future research trends," IEEE Communications Magazine, vol. 53, no. 9, pp. 74-81, 2015.

[6] S. M. R. Islam, N. Avazov, O. A. Dobre, and K. Kwak, "Power-domain non-orthogonal multiple access (NOMA) in 5G systems: Potentials and challenges," IEEE Communications Surveys Tutorials, vol. 19, no. 2, pp. 721-742, 2017.

[7] Y. Liu, Z. Qin, M. Elkashlan, Z. Ding, A. Nallanathan, and L. Hanzo, "Non orthogonal Multiple Access for 5G and Beyond," Proceedings of the IEEE, vol. 105, no. 12, pp. 2347-2381, Dec. 2017.

[8] B. Xia, J. Wang, K. Xiao, Y. Gao, Y. Yao, and S. Ma, "Outage performance analysis for the advanced SIC receiver in wireless NOMA systems," IEEE Transactions on Vehicular Technology, vol. 67, no. 7, pp. 6711-6715, Jul. 2018.
[9] T. Hou, X. Sun, and Z. Song, "Outage performance for non-orthogonal multiple access with fixed power allocation over Nakagami- $m$ fading channels," IEEE Communications Letters, vol. 22, no. 4, pp. 744-747, Apr. 2018.

[10] Y. Liu, M. Derakhshani, and S. Lambotharan, "Outage analysis and power allocation in uplink Non-Orthogonal Multiple Access systems," IEEE Communications Letters, vol. 22, no. 2, pp. 336-339, Feb. 2018.

[11] J. Wang, B. Xia, K. Xiao, Y. Gao, and S. Ma, "Outage performance analysis for wireless non-orthogonal multiple access systems," IEEE Access, vol. 6, pp. 3611-3618, Jan. 2018.

[12] L. Yang, Q. Ni, L. Lv, J. Chen, X. Xue, H. Zhang, and H. Jiang, "Cooperative non-orthogonal layered multicast multiple access for heterogeneous networks," IEEE Transactions on Communications, vol. 67, no. 2, pp. 1148-1165, Feb. 2019.

[13] F. Fang, H. Zhang, J. Cheng, S. Roy, and V. C. M. Leung, "Joint user scheduling and power allocation optimization for energy-efficient NOMA systems with imperfect CSI," IEEE Journal on Selected Areas in Communications, vol. 35, no. 12, pp. 2874-2885, Dec. 2017.

[14] Y. Gao, B. Xia, Y. Liu, Y. Yao, K. Xiao, and G. Lu, "Analysis of the dynamic ordered decoding for uplink NOMA systems with imperfect CSI," IEEE Transactions on Vehicular Technology, vol. 67, no. 7, pp. 6647-6651, Jul. 2018.

[15] F. Cui, Z. Qin, Y. Cai, M. Zhao, and G. Y. Li, "Rethinking outage constraints for resource management in NOMA networks," IEEE Journal of Selected Topics in Signal Processing, vol. 13, no. 3, pp. 1-1, Jun. 2019.

[16] D. Tweed, M. Derakhshani, S. Parsaeefard, and T. Le-Ngoc, "Outageconstrained resource allocation in uplink NOMA for critical applications," IEEE Access, vol. 5, pp. 27 636-27 648, Nov. 2017.

[17] H. Bolcskei, D. Gesbert, and A. J. Paulraj, "On the capacity of OFDMbased spatial multiplexing systems," IEEE Transactions on Communications, vol. 50, no. 2, pp. 225-234, Feb 2002.

[18] Y. Sun, D. W. K. Ng, Z. Ding, and R. Schober, "Optimal joint power and subcarrier allocation for full-duplex multicarrier non-orthogonal multiple access systems," IEEE Transactions on Communications, vol. 65, no. 3 , pp. 1077-1091, Mar. 2017.

[19] M. S. Ali, E. Hossain, A. Al-Dweik, and D. I. Kim, "Downlink power allocation for CoMP-NOMA in multi-cell networks," IEEE Transactions on Communications, vol. 66, no. 9, pp. 3982-3998, Sep. 2018

[20] L. You, D. Yuan, L. Lei, S. Sun, S. Chatzinotas, and B. Ottersten, "Resource optimization with load coupling in multi-cell NOMA," IEEE Transactions on Wireless Communications, vol. 17, no. 7, pp. 47354749, 2018.

[21] Y. Fu, Y. Chen, and C. W. Sung, "Distributed power control for the downlink of multi-cell NOMA systems," IEEE Transactions on Wireless Communications, vol. 16, no. 9, pp. 6207-6220, Sep. 2017.

[22] W. Cai, C. Chen, L. Bai, Y. Jin, and J. Choi, "User selection and power allocation schemes for downlink NOMA systems with imperfect CSI," in IEEE 84th Vehicular Technology Conference (VTC-Fall), Sep. 2016, pp. $1-5$.

[23] Z. Ding, Z. Yang, P. Fan, and H. V. Poor, "On the performance of non-orthogonal multiple access in $5 \mathrm{G}$ systems with randomly deployed users," IEEE Signal Processing Letters, vol. 21, no. 12, pp. 1501-1505, Dec 2014

[24] S. Timotheou and I. Krikidis, "Fairness for non-orthogonal multiple access in 5G systems." IEEE Signal Process. Lett., vol. 22, no. 10, pp. 1647-1651, Mar. 2015.

[25] Z. Yang, Z. Ding, P. Fan, and G. K. Karagiannidis, "On the performance of Non-orthogonal Multiple Access Systems with Partial Channel Information," IEEE Transactions on Communications, vol. 64, no. 2, pp. 654-667, Feb. 2016.

[26] S. Shi, L. Yang, and H. Zhu, "Outage balancing in downlink nonorthogonal multiple access with statistical channel state information," IEEE Transactions on Wireless Communications, vol. 15, no. 7, pp. 47184731, Jul. 2016.

[27] X. Wang, J. Wang, L. He, and J. Song, "Outage analysis for downlink NOMA with statistical channel state information," IEEE Wireless Communications Letters, vol. 7, no. 2, pp. 142-145, 2018.

[28] Z. Yang, Z. Ding, P. Fan, and Z. Ma, "Outage performance for dynamic power allocation in hybrid non-orthogonal multiple access systems," IEEE Communications Letters, vol. 20, no. 8, pp. 1695-1698, Aug. 2016.

[29] X. Yue, Z. Qin, Y. Liu, X. Dai, and Y. Chen, "Outage performance of a unified non-orthogonal multiple access framework," in IEEE International Conference on Communications (ICC), Kansas City, May 2018, pp. 1-6. 
[30] J. Cui, Z. Ding, and P. Fan, "Outage probability constrained MIMONOMA designs under imperfect CSI," IEEE Transactions on Wireless Communications, vol. 17, no. 12, pp. 8239-8255, Dec. 2018.

[31] N. Zhang, J. Wang, G. Kang, and Y. Liu, "Uplink nonorthogonal multiple access in 5G systems," IEEE Communications Letters, vol. 20, no. 3, pp. 458-461, Mar. 2016.

[32] Y. Gao, B. Xia, K. Xiao, Z. Chen, X. Li, and S. Zhang, "Theoretical analysis of the dynamic decode ordering SIC receiver for uplink NOMA systems," IEEE Communications Letters, vol. 21, no. 10, pp. 2246-2249, Oct. 2017.

[33] Z. Zhang, H. Sun, and R. Q. Hu, "Downlink and uplink non-orthogonal multiple access in a dense wireless network," IEEE Journal on Selected Areas in Communications, vol. 35, no. 12, pp. 2771-2784, Dec. 2017.

[34] J. Zeng, T. Lv, R. P. Liu, X. Su, M. Peng, C. Wang, and J. Mei, "Investigation on evolving single-carrier NOMA into multi-carrier NOMA in 5G," IEEE Access, vol. 6, pp. 48 268-48 288, Sep. 2018.

[35] Y. Sun, D. W. K. Ng, Z. Ding, and R. Schober, "Optimal joint power and subcarrier allocation for MC-NOMA systems," in IEEE Global Communications Conference (GLOBECOM), Washington, Dec. 2016, pp. 1-6.

[36] Z. Wei, D. W. K. Ng, J. Yuan, and H. Wang, "Optimal resource allocation for power-efficient MC-NOMA with imperfect channel state information," IEEE Transactions on Communications, vol. 65, no. 9, pp. 3944-3961, Sep. 2017.

[37] J. Cui, Z. Ding, and P. Fan, "A novel power allocation scheme under outage constraints in NOMA systems," IEEE Signal Processing Letters, vol. 23, no. 9, pp. 1226-1230, Sep. 2016.

[38] S. Shi, L. Yang, and H. Zhu, "Outage balancing in downlink nonorthogonal multiple access with statistical channel state information," IEEE Transactions on Wireless Communications, vol. 15, no. 7, pp. 47184731, Jul. 2016

[39] F. Yilmaz and M.-S. Alouini, "Product of Shifted Exponential Variates and Outage Capacity of Multicarrier Systems," in Wireless Conference, European. IEEE, 2009, pp. 282-286.

[40] Z. Lomnicki, "On the distribution of products of random variables," Journal of the Royal Statistical Society. Series B (Methodological), pp. 513-524, 1967.

[41] B. D. Carter and M. D. Springer, "The distribution of products, quotients and powers of independent H-function variates," SIAM Journal on Applied Mathematics, vol. 33, no. 4, pp. 542-558, 1977.

[42] R. B. Paris and D. Kaminski, Asymptotics and Mellin-Barnes Integrals. Cambridge University Press, 2001, vol. 85.

[43] M. S. Ali, H. Tabassum, and E. Hossain, "Dynamic user clustering and power allocation for uplink and downlink non-orthogonal multiple access (NOMA) systems," IEEE Access, vol. 4, pp. 6325-6343, 2016.

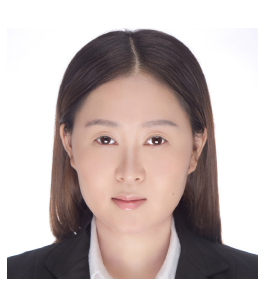

Shenhong Li (S'18) received M.Sc. degree in mobile communications from the Loughborough University, UK in 2014. She was an Intern at Nokia Bell Labs, France. She is currently working toward the Ph.D degree with the Wolfson School of Mechanical, Electrical and Manufacturing Engineering, Loughborough University, UK. Her research interests include non-orthogonal multiple access, resource allocation in wireless networks and optimization problems.

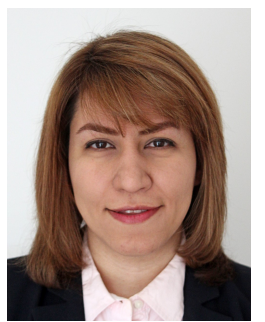

Mahsa Derakhshani (S'10, M'13) is a Lecturer (Assistant Professor) in Digital Communications with the Wolfson School of Mechanical, Electrical and Manufacturing Engineering, Loughborough University, UK. Prior to joining Lboro, she was an Honorary NSERC Postdoctoral Fellow with Department of Electrical and Electronic Engineering, Imperial College London (2015 to 2016), a Postdoctoral Research Fellow with the Department of Electrical and Computer Engineering, University of Toronto, Toronto, Canada and a Research Assistant with the Department of Electrical and Computer Engineering, McGill University (2013 to 2015). She received her Ph.D. in electrical engineering degree from McGill University, Montral, Canada, in 2013. Her research interests include radio resource management for wireless networks, software-defined wireless networking, applications of machine learning and optimization for communication systems. Dr. Derakhshani has received several awards and fellowships including the John Bonsall Porter Prize, the McGill Engineering Doctoral Award, the Fonds de Recherche du QuebecNature et Technologies (FRQNT) and Natural Sciences and Engineering Research Council of Canada (NSERC) Postdoctoral Fellowships. Currently, she serves as an Editor of IEEE Wireless Communications Letters and IET Signal Processing Journal.

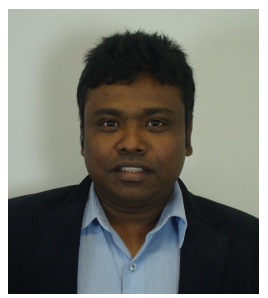

Sangarapillai Lambotharan (SM'06) received the $\mathrm{Ph} . \mathrm{D}$. degree in signal processing from Imperial College London, U.K., in 1997. He was a Visiting Scientist with the Engineering and Theory Centre, Cornell University, USA, in 1996. Until 1999, he was a Post-Doctoral Research Associate with Imperial College London. From 1999 to 2002, he was with the Motorola Applied Research Group, U.K., where he investigated various projects, including physical link layer modeling and performance characterization of GPRS, EGPRS, and UTRAN. He was with Kings College London and Cardiff University as a Lecturer and a Senior Lecturer, respectively, from 2002 to 2007. He is currently a Professor of digital communications and the Head of the Signal Processing and Networks Research Group, Wolfson School of Mechanical, Electrical and Manufacturing Engineering, Loughborough University, U.K. His current research interests include 5G networks, MIMO, blockchain, machine learning, and network security. He has authored more than 200 journal articles and conference papers in these areas. He serves as an Associate Editor for IEEE TRANSACTIONS ON SIGNAL PROCESSING

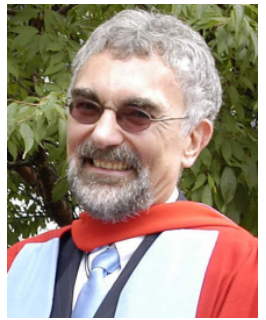

Lajos Hanzo (http://www-mobile.ecs.soton.ac.uk, https://en.wikipedia.org/wiki/Lajos_Hanzo) FREng, FIEEE, FIET, Fellow of EURASIP, DSc holds an honorary doctorate by the Technical University of Budapest (2009) and by the University of Edinburgh (2015). He is a Foreign Member of the Hungarian Academy of Sciences and a former Editor-in-Chief of the IEEE Press. He has served as Governor of both IEEE ComSoc and of VTS. He has published $1900+$ contributions at IEEE Xplore, 18 Wiley-IEEE Press books and has helped the fast-track career of $119 \mathrm{PhD}$ students. Over 40 of them are Professors at various stages of their careers in academia and many of them are leading scientists in the wireless industry. 